\title{
$\mathrm{NAD}^{+}$homeostasis in renal health and disease
}

Kenneth M. Ralto' ${ }^{1}$ Eugene P. Rhee ${ }^{2}$ and Samir M. Parikh (1) ${ }^{3 *}$

Abstract | The mammalian kidney relies on abundant mitochondria in the renal tubule to generate sufficient ATP to provide the energy required for constant reclamation of solutes from crude blood filtrate. The highly metabolically active cells of the renal tubule also pair their energetic needs to the regulation of diverse cellular processes, including energy generation, antioxidant responses, autophagy and mitochondrial quality control. Nicotinamide adenine dinucleotide $\left(\mathrm{NAD}^{+}\right)$is essential not only for the harvesting of energy from substrates but also for an array of regulatory reactions that determine cellular health. In acute kidney injury (AKI), substantial decreases in the levels of $\mathrm{NAD}^{+}$impair energy generation and, ultimately, the core kidney function of selective solute transport. Conversely, augmentation of $\mathrm{NAD}^{+}$may protect the kidney tubule against diverse acute stressors. For example, $\mathrm{NAD}^{+}$augmentation can ameliorate experimental AKI triggered by ischaemia-reperfusion, toxic injury and systemic inflammation. $\mathrm{NAD}^{+}$-dependent maintenance of renal tubular metabolic health may also attenuate long-term profibrotic responses that could lead to chronic kidney disease. Further understanding of the genetic, environmental and nutritional factors that influence $\mathrm{NAD}^{+}$biosynthesis and renal resilience may lead to novel approaches for the prevention and treatment of kidney disease.

'Division of Nephrology and Department of Medicine, UMass Memorial Medical Center and University of Massachusetts Medical School, Worcester, MA, USA. ${ }^{2}$ Division of Nephrology and Department of Medicine, Massachusetts General Hospital and Harvard Medical School, Boston, MA, USA.

${ }^{3}$ Division of Nephrology, Center for Vascular Biology Research and Department of Medicine, Beth Israel Deaconess Medical Center and Harvard Medical School, Boston, MA, USA.

*e-mail:sparikh1@ bidmc.harvard.edu https://doi.org/10.1038/ s41581-019-0216-6
Nicotinamide adenine dinucleotide $\left(\mathrm{NAD}^{+}\right)$is found in all known forms of life. As a carrier of high-energy electrons from fuel oxidation (in its reduced form, NADH), $\mathrm{NAD}^{+}$is an essential cofactor for redox reactions in the cytosol and mitochondria. These redox reactions are critical in enabling the most basic function of the cell, namely, the harvesting of energy in the form of ATP from fuel substrates such as glucose, amino acids and fatty acids. Without $\mathrm{NAD}^{+}$, fuel cannot be converted into energy. As a substrate for major classes of enzymes such as sirtuins that cleave a carbon-nitrogen bond in $\mathrm{NAD}^{+}$to yield nicotinamide (Nam; also known as niacinamide) and ADPribose (ADPR), $\mathrm{NAD}^{+}$also regulates a variety of other cellular processes, including the cellular stress response, mitochondrial homeostasis and calcium signalling.

During the past 20 years, $\mathrm{NAD}^{+}$has garnered substantial interest as a target in ageing research. In this context, $\mathrm{NAD}^{+}$has been most extensively investigated as a substrate for sirtuins, which act in the nucleus, cytosol and mitochondria to deacetylate or deacylate target proteins, including DNA histones, DNA repair enzymes and transcriptional regulators of metabolism. Over the past 3 years, data from experimental models and clinical studies have also implicated $\mathrm{NAD}^{+}$homeostasis as a determinant of kidney health and of the ability of the renal tubule to resist varied stressors. Here, we review fundamental aspects of $\mathrm{NAD}^{+}$biology and discuss evidence for a role of $\mathrm{NAD}^{+}$imbalance in the pathogenesis of acute kidney injury (AKI) and chronic kidney disease (CKD). Finally, we propose that disruption of $\mathrm{NAD}^{+}$metabolism may contribute to mechanistic links among AKI, CKD and ageing.

\section{NAD ${ }^{+}$homeostasis}

$\mathrm{NAD}^{+}$metabolism is a highly dynamic process and all four NAD coenzymes - NAD ${ }^{+}, \mathrm{NADH}, \mathrm{NADP}^{+}$and $\mathrm{NADPH}$ - have redox and signalling functions (FIC. 1). $\mathrm{NAD}^{+}$serves as a cofactor for most enzymes that oxidize substrates and is itself reduced to NADH. Conversely, NADPH often functions as a cofactor for enzymes that reduce substrates. Thus, $\mathrm{NAD}^{+}$homeostasis has key roles in cellular catabolism and anabolism. In addition, $\mathrm{NAD}^{+}$ can undergo cleavage to generate ADPR or signalling molecules, act as an acceptor for acyl and acetyl modifications removed from proteins via sirtuins and undergo phosphorylation to form $\mathrm{NADP}^{+}$.

\section{$N A D^{+}$and NADH redox cycling}

$\mathrm{NAD}^{+}$is an electron carrier for oxidative reactions in the cytosol and mitochondria. These reactions result in a continuous redox cycle of reduction to NADH and oxidation back to $\mathrm{NAD}^{+}$. This $\mathrm{NAD}^{+}$recycling is required for the continuous flow of electrons across intermediary metabolism, including the oxidation of fuel 


\section{Key points \\ - $\mathrm{NAD}^{+}$has critical roles in the generation of ATP from fuel substrates and as a substrate for important enzymes that regulate cellular health and stress responses. \\ - The renal tubule is highly metabolically active and requires a constant supply of ATP to provide the energy required to pump solutes across unfavourable gradients. \\ - Experimental acute kidney injury (AKI) induced by various insults rapidly leads to a decrease in $\mathrm{NAD}^{+}$levels that probably results from a combination of reduced $\mathrm{NAD}^{+}$ biosynthesis and increased $\mathrm{NAD}^{+}$consumption. \\ - Renal NAD+ levels can be augmented using vitamin $\mathrm{B}_{3}$ analogues and related nutritional precursors. \\ - $\mathrm{NAD}^{+}$augmentation can prevent and/or treat various aetiologies of experimental AKI and might also attenuate long-term profibrotic responses following AKI, suggesting a potential role in the treatment of chronic kidney disease.}

substrates via glycolysis, the tricarboxylic acid (TCA) cycle (also known as the citric acid cycle and Krebs cycle) and $\beta$-oxidation of fatty acids. For example, the oxidation of glyceraldehyde-3-phosphate to 1,3-bisphosphoglycerate in glycolysis is coupled to the reduction of cytosolic $\mathrm{NAD}^{+}$to NADH. The oxidized form, $\mathrm{NAD}^{+}$, must be regenerated and available for subsequent reactions. Under aerobic conditions, the transfer of electrons into mitochondria via the malate-aspartate shuttle or glycerol-3-phosphate shuttle, and subsequently to the mitochondrial electron transport chain (ETC), regenerates cytosolic $\mathrm{NAD}^{+}$. Mitochondrial NADH produced during the TCA cycle and $\beta$-oxidation can donate electrons directly to the ETC. When mitochondrial respiration is impaired, the cytosolic reduction of pyruvate to lactate regenerates $\mathrm{NAD}^{+}$to permit ongoing glycolysis.

\section{NAD biosynthesis}

In addition to redox cycling, $\mathrm{NAD}^{+}$undergoes net biosynthesis and consumption. $\mathrm{NAD}^{+}$can be synthesized from several distinct dietary precursors via three separate pathways (FIG. 2). The de novo pathway converts dietary tryptophan to $\mathrm{NAD}^{+}$via eight sequential steps. Early metabolites in this pathway can participate in the generation of diverse molecules, including melatonin and the inflammatory signalling molecule kynurenine; however, a key step catalysed by quinolinate phosphoribosyltransferase (QPRT) commits the pathway to $\mathrm{NAD}^{+}$ biosynthesis. QPRT has an important role in maintaining renal $\mathrm{NAD}^{+}$levels

Four other dietary compounds - Nam, nicotinamide riboside (NR), nicotinamide mononucleotide $(\mathrm{NMN})$ and niacin (the acid form of vitamin $\mathrm{B}_{3}$; also known as nicotinic acid) - can also serve as $\mathrm{NAD}^{+}$precursors. Nam is the precursor of the salvage pathway ${ }^{2,3}$, the name of which relates to Nam being the product of $\mathrm{NAD}^{+}$consumption reactions. NR flows into the salvage pathway and both Nam and NR are converted to the penultimate product of this pathway, NMN, which may also be directly absorbed from the gut ${ }^{4}$. Niacin is the precursor in the Preiss-Handler pathway. The redundancy in these biosynthetic pathways is illustrated by pellagra, which is caused by a lack of niacin that results in $\mathrm{NAD}^{+}$ deficiency. Pellagra classically leads to diarrhoea, dermatitis, dementia and, in severe cases, death. Adequate dietary intake of either tryptophan or niacin is sufficient to prevent this disease ${ }^{5}$.
The salvage pathway is responsible for most $\mathrm{NAD}^{+}$ production in mammalian cells ${ }^{2,3}$. As mentioned above, supplementation with tryptophan - the first substrate of the de novo pathway - has long been recognized as a treatment for vitamin $B_{3}$ deficiency, suggesting an important role for the de novo pathway in $\mathrm{NAD}^{+}$homeostasis 6 . However, radioisotope studies conducted in the 1970s showed that Nam was the major $\mathrm{NAD}^{+}$precursor in the mouse liver?. In 2018, a quantitative analysis of $\mathrm{NAD}^{+}$synthesis and breakdown in mice that used flux studies and mathematical modelling reported that the oral bioavailability of exogenous Nam was greater than that of NR and NMN; however, the de novo pathway from tryptophan substantially contributed to baseline $\mathrm{NAD}^{+}$production in the liver and kidney ${ }^{8}$. Another study suggested that NR was more effective at increasing intracellular $\mathrm{NAD}^{+}$levels than Nam or nicotinic acid'. Moreover, a pilot trial showed that NR supplementation increased $\mathrm{NAD}^{+}$levels in the peripheral blood mononuclear cells of healthy adults ${ }^{10}$. Among these $\mathrm{NAD}^{+}$precursors, only the ingestion of niacin is associated with a transient vasodilatory flushing reaction ${ }^{11}$.

\section{$N A D^{+}$consumption}

$\mathrm{NAD}^{+}$consumption is catalysed by three classes of enzymes: sirtuins, poly(ADP-ribose) polymerases (PARPs) and cyclic ADPR (cADPR) synthetases (FIG. 3).

Sirtuins. Sirtuins are a family of proteins that are evolutionarily conserved from yeast. Seven different sirtuins have been identified in mammals, each with distinct cellular localization and function. For example, SIRT1 is primarily located in the nucleus where it is associated with euchromatin, whereas SIRT3-5 are primarily mitochondrial ${ }^{12}$. Sirtuins are involved in many reactions that regulate cellular metabolism through the $\mathrm{NAD}^{+}$-dependent deacetylation or deacylation of proteins including histones, such as $\mathrm{H} 3, \mathrm{H} 4$ and $\mathrm{H} 1$, transcription factors and co-activators, including p53, NF- $\mathrm{KB}$, PPAR $\gamma$ co-activator $1 \alpha(\mathrm{PGCl} \alpha)$ and FOXO1, and signalling regulators of metabolism, including protein kinase $\mathrm{A}$, AMP-activated protein kinase (AMPK) and mechanistic target of rapamycin $(\mathrm{mTOR})^{13}$. Sirtuins also utilize $\mathrm{NAD}^{+}$ to remove other kinds of post-translational protein modifications, including malonylation and succinylation. In all of these reactions, the ADPR generated from $\mathrm{NAD}^{+}$ becomes an acceptor for the post-translational group removed from the target protein. For example, during the deacetylation of histones, SIRT1 transfers the acetyl group from key lysine residues of the histone protein to ADPR. Sirtuin activity is increased with caloric restriction and has been associated with increased longevity and/or health in yeast, worms, flies and mice ${ }^{14-17}$; however, these links have been questioned, as other studies have reported that lifespan extension in yeast, worms and flies may be sirtuin-independent ${ }^{18}$.

Poly(ADP-ribose) polymerases. PARPs utilize NAD ${ }^{+}$ as a substrate to attach one or more ADPRs to target proteins in response to cellular stress, particularly DNA damage. PARP-dependent ADP-ribosylation of proteins that interact with DNA, such as histones, is considered to 


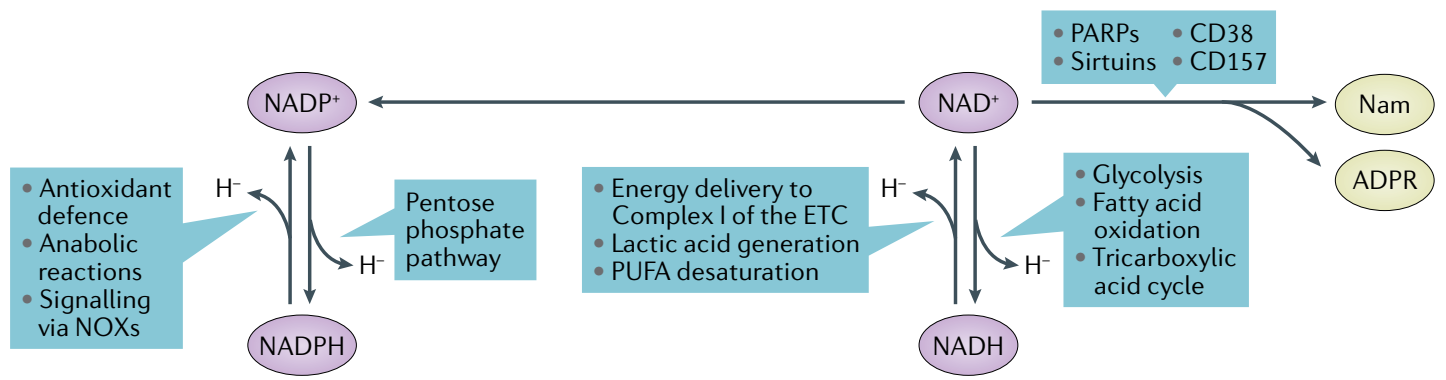

Fig. 1 | Overview of $\mathrm{NAD}^{+}$metabolism In highly metabolically active mammalian cells such as the renal tubular epithelium, nicotinamide adenine dinucleotide (NAD+) acts as a hub that coordinates various aspects of metabolism. Addition of a hydride ion $\left(\mathrm{H}^{-}\right)$(an electron pair) to $\mathrm{NAD}^{+}$yields its reduced form, NADH. Reduction of NAD ${ }^{+}$ to $\mathrm{NADH}$ is required for glycolysis, fatty acid oxidation and the tricarboxylic acid cycle (also known as the citric acid cycle and Krebs cycle). Oxidation of NADH (donation of the electron pair) moves high-energy electrons from fuel substrates to Complex I of the mitochondrial electron transport chain (ETC). Sequential oxidation-reduction reactions in the ETC generate a proton motive force that is utilized by mitochondrial ATP synthase to generate ATP (not shown). Oxidation of $\mathrm{NADH}$ is also required to generate lactic acid from pyruvate and for the desaturation of polyunsaturated fatty acids (PUFAs). $\mathrm{NAD}^{+}$can also be phosphorylated to form NADP ${ }^{+}$. Oxidation-reduction reactions involving $\mathrm{NADP}^{+}$and its reduced form, NADPH, facilitate defence against oxidative stress by serving as a cofactor for thioredoxin-dependent reduction of disulfides and as a cofactor for glutathione reductase, which reduces the disulfide form of glutathione to its antioxidant sulfhydryl form. Reduction of $\mathrm{NADP}^{+}$also occurs in the pentose phosphate pathway, which generates chemical building blocks for sugars and nucleic acids. NADPH serves as a substrate for NADPH oxidase enzymes (NOXs) that create second messengers of free radicals. $\mathrm{NAD}^{+}$can also be cleaved by poly(ADP-ribose) polymerases (PARPs), sirtuins and the cyclic ADP-ribose (ADPR) synthetases CD38 and CD157 to generate nicotinamide (Nam) and ADPR.

be a protective mechanism in the setting of mild cellular injury. However, the intense PARP activation that occurs with severe insults may also lead to $\mathrm{NAD}^{+}$depletion and cell death. A PARP1 inhibitor, olaparib, has been FDAapproved for the treatment of breast and ovarian cancers linked to mutations that promote DNA damage.

Cyclic ADP-ribose synthetases. CADPR synthetases are classically activated during inflammation and hydrolyse a variety of nucleotide metabolites, including ATP and ADP. Two of these enzymes - CD38 and its homologue $\mathrm{CD} 157$ - cleave $\mathrm{NAD}^{+}$and have been associated with an age-related decrease in $\mathrm{NAD}^{+}$levels ${ }^{19,20}$. CD38 hydrolyses $\mathrm{NAD}^{+}$to generate Nam and several different secondmessenger molecules, including cADPR. A smallmolecule inhibitor of CD38 has been shown to increase tissue $\mathrm{NAD}^{+}$levels and promote metabolic changes associated with increased longevity in aged mice ${ }^{21}$.

\section{NAD ${ }^{+}$compartmentalization}

As several pathways are involved in $\mathrm{NAD}^{+}$synthesis and consumption, the intracellular pool of $\mathrm{NAD}^{+}$is constantly in flux. The half-life of $\mathrm{NAD}^{+}$is $1-2 \mathrm{~h}$ in the cytoplasm and nucleus and approximately $8 \mathrm{~h}$ in mitochondria ${ }^{22,23}$. Despite this short half-life, the intracellular concentration of $\mathrm{NAD}^{+}$is thought to remain fairly constant in healthy states, varying only a few-fold with changes in metabolic activity ${ }^{24}$. Elegant studies using a $\mathrm{NAD}^{+}$biosensor showed that $\mathrm{NAD}^{+}$concentration varies substantially between different intracellular compartments ${ }^{23}$. The cytoplasm and nucleus of HEK293T cells had free $\mathrm{NAD}^{+}$concentrations of approximately $100 \mu \mathrm{M}$, which was approximately half that of mitochondria. These three pools of $\mathrm{NAD}^{+}$may be connected, although a mammalian intracellular $\mathrm{NAD}^{+}$transporter has not yet been identified ${ }^{23}$. If $\mathrm{NAD}^{+}$concentrations are regulated in a compartment-specific manner, this regulation could have an impact on the redox function to generate ATP as well as the substrate function for $\mathrm{NAD}^{+}$-consuming enzymes. For example, a reduction in the level of PARP1, which is predominantly localized in the nucleus, markedly increased the nuclear:cytoplasmic ratio of $\mathrm{NAD}^{+}$, without altering mitochondrial $\mathrm{NAD}^{+}$levels or the activity of mitochondrial SIRT3 (REF. ${ }^{25}$ ).

\section{$\mathrm{NADP}^{+}$and NADPH}

Redox cycling, biosynthesis and consumption of $\mathrm{NAD}^{+}$ are all closely linked to the cellular balance of $\mathrm{NADP}^{+}$ (FIG. 1). As mentioned above, $\mathrm{NADP}^{+}$can be generated directly by phosphorylation of $\mathrm{NAD}^{+}$via $\mathrm{NAD}^{+}$kinases, whereas transfer of a hydride from $\mathrm{NADH}$ to $\mathrm{NADP}^{+}$via Nam-nucleotide transhydrogenase generates NADPH. Conversion of $\mathrm{NAD}^{+}$to $\mathrm{NADP}^{+}$is estimated to consume approximately $10 \%$ of cellular $\mathrm{NAD}^{+}\left(\mathrm{REF}^{8}\right)^{8}$. $\mathrm{NADP}^{+}$can also be generated from Nam via $\mathrm{NAD}(\mathrm{P})$ nucleosidase, which catalyses the reaction between Nam and ADPR phosphate.

$\mathrm{NADPH}$ is generated from $\mathrm{NADP}^{+}$by the pentose phosphate pathway, which operates in parallel with glycolysis in the cytosol and is responsible for approximately $10-20 \%$ of glucose utilization. NADPH has an important role in antioxidant defence and serves several reductive and signalling functions in the cell. Fatty acid synthases utilize NADPH as a cofactor to synthesize fatty acids from acetyl-CoA; NADPH oxidases generate oxygen free radicals from NADPH that are used by neutrophils for the oxidative burst during antimicrobial defence; and glutathione reductase uses NADPH to catalyse the reduction of glutathione disulfide to glutathione ${ }^{26}$. Another major function of the pentose phosphate pathway is to generate metabolic building blocks, such as ribose-5-phosphate and erythrose-4-phosphate, 
Mitochondrial biogenesis The process by which cells increase the mass of mitochondria.

Mitophagy

The process of intracellular degradation of mitochondria by autophagy. which are used in nucleic acid and aromatic amino acid synthesis, respectively ${ }^{27}$.

To meet the changing needs of metabolically active cells, $\mathrm{NADP}^{+}$can be converted back to $\mathrm{NAD}^{+}$by $\mathrm{NADP}^{+}$ phosphatases ${ }^{28}$. $\mathrm{NADP}^{+}$is present in cells at an approximately 20 -fold lower concentration and has a shorter half-life than $\mathrm{NAD}^{+}\left(\mathrm{REF}^{8}\right)$. As NADPH has roles in the generation of vascular protective factors and protection against oxidative stress ${ }^{29,30}$, this small pool of dinucleotides may also have an impact on renal health and disease.

\section{Modulation of metabolism by NAD ${ }^{+}$}

As a substrate for the consumptive reactions described above, $\mathrm{NAD}^{+}$has a key role in cellular metabolism and signalling. For example, $\mathrm{NAD}^{+}$-dependent activation of SIRT1 leads to SIRT1-mediated deacetylation and activation of the transcriptional co-activator of mitochondrial biogenesis, PGC1a, which results in enhanced lipid oxidation and ATP production. Activation of AMPK signalling can also activate PGC1a via phosphorylation. SIRT1 and AMPK can also regulate each other; SIRT1 can activate AMPK by signalling

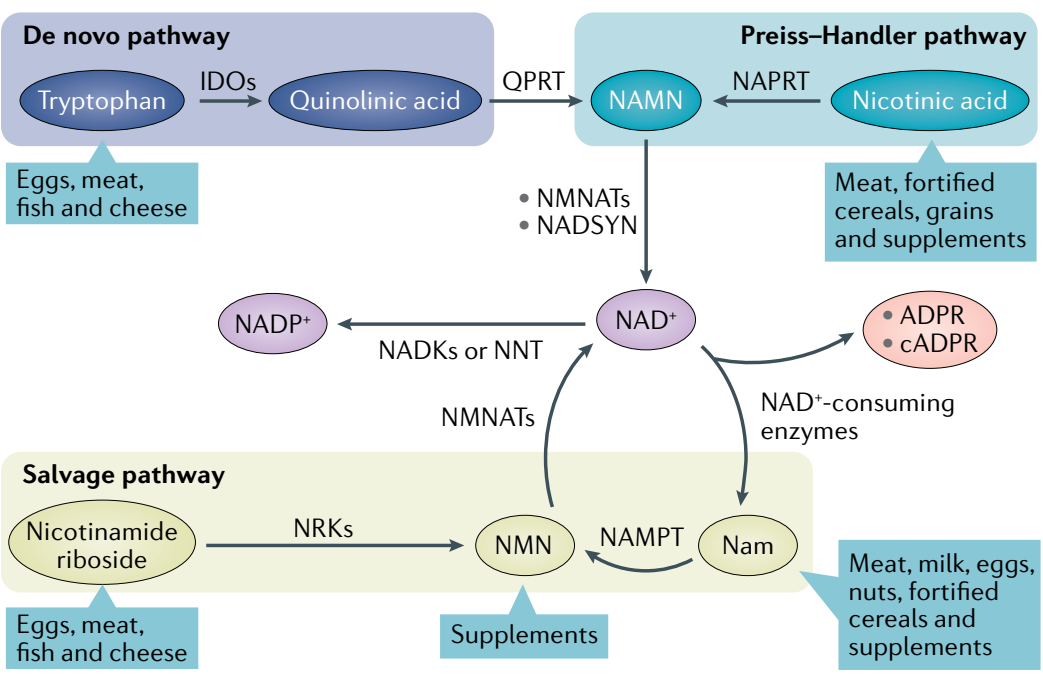

Fig. 2 | Pathways of $\mathrm{NAD}^{+}$biosynthesis Dietary nutrients are converted to nicotinamide adenine dinucleotide (NAD+) through discrete endogenous biosynthetic routes. The de novo pathway converts tryptophan to $\mathrm{NAD}^{+}$via a series of enzymatic steps. Indoleamine-2,3-dioxygenase (IDO) is a rate-limiting enzyme that catalyses the first step in this pathway, the conversion of tryptophan to $\mathrm{N}$-formylkynurenine (not shown). Quinolinate phosphoribosyltransferase (QPRT) catalyses the conversion of quinolinic acid to nicotinate mononucleotide (NAMN). This bottleneck step commits the pathway to $\mathrm{NAD}^{+}$synthesis. The Preiss-Handler pathway converts the acid form of vitamin $\mathrm{B}_{3}$, nicotinic acid (also known as niacin), to NAMN. This conversion is catalysed by nicotinate phosphoribosyltransferase (NAPRT). NAMN is converted to NAD ${ }^{+}$by the sequential action of nicotinamide mononucleotide adenylyl transferases (NMNATs) and NAD synthetase (NADSYN). The salvage pathway converts the base analogue of vitamin $B_{3}$, nicotinamide (Nam; also known as niacinamide), to $\mathrm{NAD}^{+}$via the rate-limiting enzyme Nam phosphoribosyltransferase (NAMPT). Nam mononucleotide (NMN) is a measurable intermediate in this pathway that can also be obtained through supplements and synthesized from the dietary precursor nicotinamide riboside. The salvage pathway is so named because $\mathrm{NAD}^{+}$is 'reclaimed' from the Nam product of enzymes that consume $\mathrm{NAD}^{+}$to generate ADP-ribose (ADPR) or cyclic ADPR (cADPR). NAD ${ }^{+}$is converted to $\mathrm{NAD}^{+}$phosphate $\left(\mathrm{NADP}^{+}\right)$by $\mathrm{NAD}^{+}$kinases (NADKs). Hydride transfer from NADH to $\mathrm{NADP}^{+}$by nicotinamide nucleotide transhydrogenase (NNT) also generates NADPH. NRKs, nicotinamide riboside kinases. through liver kinase B1 (LKB1), whereas AMPK can promote SIRT1 activity by increasing the availability of NAD ${ }^{+}\left(\right.$REF. $\left.^{31}\right)$. When SIRT1, AMPK and PGC1a are active, gene transcription shifts to promote mitochondrial function and oxidative metabolism. Together, SIRT1, AMPK and PGC1a comprise an energy-sensing network downstream of $\mathrm{NAD}^{+}$that is fundamental to cellular energy expenditure ${ }^{32}$.

$\mathrm{NAD}^{+}$-mediated SIRT1 activation also leads to increased transcription and translation of mitochondrial DNA-encoded but not nuclear DNA-encoded components of the $\mathrm{ETC}^{33}$. The resulting imbalance can trigger the mitochondrial unfolded protein response $\left(\mathrm{UPR}^{\mathrm{mt}}\right)$, which synchronizes the activity of the mitochondrial and nuclear genomes to maintain a healthy mitochondrial proteome. The $\mathrm{UPR}^{\mathrm{mt}}$ involves a range of processes, including the upregulation of chaperone proteins, proteases, antioxidant enzymes and mitophagy ${ }^{34}$.

Importantly, $\mathrm{NAD}^{+}$consumption by PARPs and $\mathrm{CD} 38$ can reduce its availability for consumption by sirtuins, and thus shift metabolic signalling in the cell. For example, PARP enzymes respond to DNA damage by utilizing $\mathrm{NAD}^{+}$to generate PAR polymers on proteins. This PARP activity is sufficiently robust to attenuate SIRT1 activity as a direct result of local $\mathrm{NAD}^{+}$depletion $^{35}$. Conversely, PARP inhibition increases mitochondrial metabolism through SIRT activation in an $\mathrm{NAD}^{+}$-dependent manner ${ }^{25}$. Through these and additional interactions reviewed in detail elsewhere, PARPs and sirtuins may have counterbalancing roles in the regulation of cellular metabolism ${ }^{36}$.

\section{NAD ${ }^{+}$and kidney disease}

Energy metabolism in the nephron involves both aerobic and anaerobic generation of ATP and is exquisitely adapted to the constraints of blood flow and countercurrent exchange. For example, the hypoxic environment of the inner medulla is matched by a near absence of mitochondria within those tubular cells. Even in the welloxygenated cortex, different segments of the proximal tubule preferentially utilize different fuel substrates ${ }^{37}$. Fatty acids may be a chief fuel source for the S1 and S2 segments of the proximal tubule, whereas the less welloxygenated straight or S3 segment may also utilize glu$\operatorname{cose}^{38}$. Despite these important regional variations, the kidney is second only to the heart in the abundance of mitochondria ${ }^{39}$. Rare monogenic human diseases provide the clearest evidence that intact mitochondria are required for renal health ${ }^{40}$. An essential role for $\mathrm{NAD}^{+}$ biosynthesis within this larger framework is demonstrated by the identification of mutations in two de novo $\mathrm{NAD}^{+}$biosynthesis genes, KYNU and $H A A O$, in patients with multiple congenital malformations, including dysplastic kidneys ${ }^{41}$.

\section{Acute kidney injury}

Most of the renal $\mathrm{NAD}^{+}$research reported to date has used models of tubular biology. The cellular stress that is associated with different AKI syndromes triggers accelerated consumption of $\mathrm{NAD}^{+}$by PARP enzymes ${ }^{42,43}$. Emerging evidence suggests that inter-individual differences, both in risk of AKI and in outcomes following 

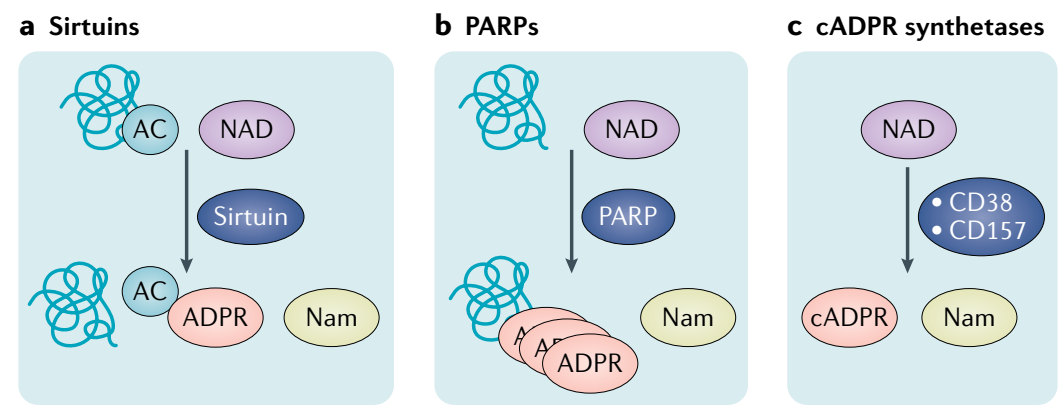

Fig. 3 | NAD ${ }^{+}$-consuming enzymes. Nicotinamide adenine dinucleotide (NAD ${ }^{+}$consuming enzymes comprise sirtuins (part a), poly(ADP-ribose) polymerases (PARPs) (part b) and the cyclic ADP-ribose (cADPR) synthetases CD38 and CD157 (part c). Sirtuins deacetylate or deacylate proteins by cleaving $N A D^{+}$to nicotinamide (Nam) and ADPR, which accepts the acetyl or acyl group (AC) from the target protein. PARPs utilize $\mathrm{NAD}^{+}$cleavage to attach single or multiple ADPR subunits to target proteins. CD38 and $\mathrm{CD} 157$ cleave $\mathrm{NAD}^{+}$to form cADPR and Nam.

AKI, may relate to differences in the ability to buffer the stress-associated consumption of tubular $\mathrm{NAD}^{+}$with $\mathrm{NAD}^{+}$biosynthesis.

Accelerated $\mathrm{NAD}^{+}$consumption. $\mathrm{NAD}^{+}$-consuming PARPs are pathologically induced in the setting of AKI. In rats subjected to renal ischaemia-reperfusion injury (IRI), tubular PARP expression was increased 6-12 h after reperfusion ${ }^{42}$. Systemic administration of a benzamide-based PARP inhibitor after reperfusion increased renal levels of ATP, reduced histopathological evidence of tubular damage and improved renal function in these rats. Consistent with these findings, Parp1-knockout mice were more resistant to renal IRI than wild-type controls ${ }^{44}$. Subsequent studies showed that a PARP1 inhibitor preserved renal blood flow in a rat model of ventilator-induced lung injury ${ }^{45}$ and that intravenous benzamide administration prevented a decline in renal blood flow in dogs subjected to endotoxaemic shock ${ }^{43}$. A study published in abstract form also reported beneficial effects of a novel PARP1 inhibitor in a rat model of renal IRI ${ }^{46}$. Taken together, these results implicate accelerated $\mathrm{NAD}^{+}$consumption owing to induction of PARPs in the development of AKI. Several PARP inhibitors, including olaparib, rucaparib and talazoparib, have been FDA-approved for the treatment of BRCA-related breast and ovarian cancer, making PARP inhibition tractable for clinical evaluation in AKI.

Defective de novo $\mathrm{NAD}^{+}$biosynthesis. The kidney possesses the enzymatic machinery to synthesize $\mathrm{NAD}^{+}$ by all of the known mammalian pathways. Evidence that de novo $\mathrm{NAD}^{+}$biosynthesis is impaired in $\mathrm{AKI}$ emerged from studies aimed at identifying downstream effectors of the renoprotective effects of PGC1 $\alpha^{1,47}$. In cultured renal tubular cells, overexpression of PGC1a protected against oxidant stress ${ }^{48}$. Our research group found that mice with global or tubule-specific knockout of the gene encoding PGC1 1 exhibited blunted recovery and worse renal injury following endotoxaemia, systemic cisplatin treatment or bilateral renal IRI compared with wild-type controls ${ }^{47,49,50}$. Conversely, mice with tubule-specific overexpression of PGC1 1 were protected against all three models of $\mathrm{AKI}^{47,49,50}$. The PPARGC1A locus, which encodes PGC1a, contains an evolutionarily conserved upstream open reading frame (uORF) in the $5^{\prime}$ untranslated region ${ }^{51}$. Such uORFs reduce protein expression by suppressing translation ${ }^{52}$. Using CRISPR-Cas9, we mutated the start codon of the Ppargcla uORF to create a transgenic mouse with global overexpression of PGC1 $\alpha$ and showed that this mouse was protected against renal IRI ${ }^{51}$. The available data obtained using gain-of-function and lossof-function mice in distinct models of AKI indicate that PGC1 $\alpha$ may be a common and conserved determinant of the injury response of tubular cells to many different stressors.

To identify downstream effectors of the renoprotective actions of PGC1 1 , we conducted metabolomics analysis and RNA sequencing of kidneys from PGC1a loss-of-function and gain-of-function mice with and without IRI ${ }^{47}$. We found that IRI suppressed the renal expression of enzymes in the de novo $\mathrm{NAD}^{+}$biosynthesis pathway and was associated with reduced levels of Nam and $\mathrm{NAD}^{+}$. Ppargcla-knockout mice, which exhibited increased sensitivity to IRI, had basal reductions in the expression of de novo $\mathrm{NAD}^{+}$biosynthesis enzymes and in the renal levels of $\mathrm{Nam}$ and $\mathrm{NAD}^{+}$. Conversely, mice with gain of function of PGC1 $\alpha$, which developed less severe AKI and recovered more quickly following IRI than littermate controls, showed a basal induction of the de novo $\mathrm{NAD}^{+}$biosynthesis pathway and elevated renal levels of Nam and $\mathrm{NAD}^{+}$compared with these controls. Notably, renal tubular PGC1a overexpression did not modulate expression of PARPs, sirtuins, CADPR synthetases or key enzymes in other $\mathrm{NAD}^{+}$ biosynthetic pathways. These results demonstrate that PGC1 $\alpha$ in the renal tubule coordinates the biosynthesis of $\mathrm{NAD}^{+}$through the de novo pathway. Considered in the context of earlier results showing that $\mathrm{NAD}^{+} \mathrm{can}$ post-translationally activate PGC1 $\alpha^{53}$, the findings suggest that $\mathrm{PGCl} \alpha$ and $\mathrm{NAD}^{+}$might reciprocally regulate each other.

A metabolomics analysis of mouse urine samples identified an increase in urinary quinolinic acid following IRI ${ }^{1}$. Unlike upstream metabolites in the de novo $\mathrm{NAD}^{+}$biosynthesis pathway, the only known function of quinolinic acid is to serve as a precursor for $\mathrm{NAD}^{+}$ (FIG. 4). Accumulation of quinolinic acid in the postischaemic urine suggested a reduction in the levels of the enzyme that utilizes this substrate, QPRT. As predicted by the metabolomics findings and reported in a previous study ${ }^{47}$, the renal expression of QPRT was reduced in post-ischaemic kidneys. Compared with wild-type littermates, mice that were heterozygous for QPRT recapitulated nearly half of the AKI urinary metabolomic pattern, even in the absence of AKI. QPRT heterozygous mice were more susceptible to renal IRI than wild-type littermate controls; however, this increased susceptibility was abrogated by augmenting $\mathrm{NAD}^{+}$biosynthesis via the salvage pathway using exogenous Nam. These results suggest that AKI-induced suppression of QPRT might be a pathogenic event in AKI that can be addressed by orthogonal replenishment of $\mathrm{NAD}^{+}$. 


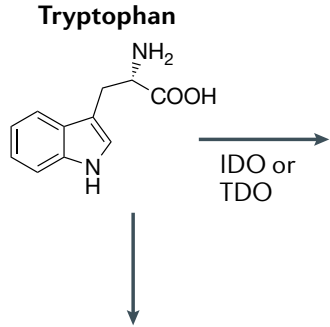

- Tryptamine

- Indole-3-

acetaldehyde

5OH-tryptophan

5OH-kynurenamine

- Serotonin

Melatonin

5OH-indoleacetate
$N$-formylkynurenine

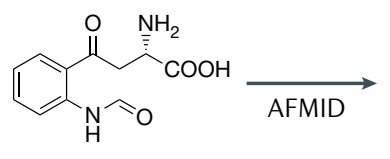

Kynurenine

$\mathrm{KMO}$

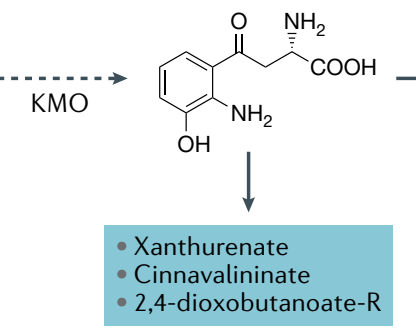

3-hydroxyl-kynurenine

3-hydroxyanthranilic acid
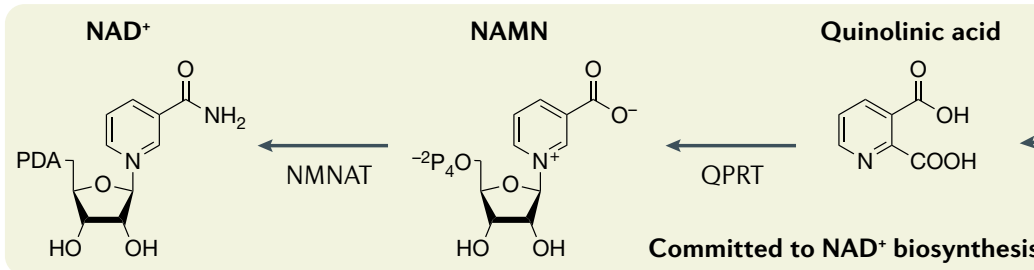

Committed to $\mathrm{NAD}^{+}$biosynthesis

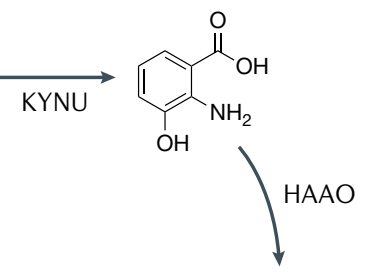

ACMS

Fig. 4 | The de novo NAD ${ }^{+}$biosynthesis pathway. The de novo nicotinamide adenine dinucleotide (NAD ${ }^{+}$) biosynthesis pathway (also known as the kynurenine pathway or tryptophan-kynurenine pathway) consists of eight steps that convert dietary tryptophan to NAD+. Tryptophan has multiple metabolic fates that compete with its conversion to $\mathrm{N}$-formylkynurenine, which is catalysed by indoleamine 2,3-dioxygenase (IDO) or Trp 2,3-dioxygenase (TDO) ${ }^{110}$. $\mathrm{N}$-formylkynurenine is hydrolysed to kynurenine by arylformamidase (AFMID). Kynurenine is hydroxylated to 3-hydroxykynurenine by kynurenine 3-monooxygenase (KMO). 3-Hydroxy-kynurenine is a major branch point in the pathway as this metabolite can be converted to xanthurenate or other molecules. Kynureninase (KYNU) catalyses the formation of 3-hydroxyanthranilic acid, which is converted to $\alpha$-amino- $\beta$-carboxymuconate- $\varepsilon$-semialdehyde (ACMS) by 3-hydroxyanthranilate 3,4-dioxygenase (HAAO). ACMS can either spontaneously cyclize to form quinolinic acid or can be decarboxylated by ACMS decarboxylase (ACMSD) to form picolinic acid. The only known fate of quinolinic acid is conversion to nicotinate mononucleotide (NAMN) by quinolinate phosphoribosyltransferase (QPRT). Quinolinic acid is therefore the first metabolite in the pathway that is committed to NAD ${ }^{+}$biosynthesis. Nicotinamide mononucleotide adenylyltransferase (NMNAT) catalyses the final step to create NAD+.

In the de novo pathway of $\mathrm{NAD}^{+}$biosynthesis, the intermediate amino- $\beta$-carboxymuconate- $\varepsilon$-semialdehyde (ACMS) spontaneously cyclizes to form quinolinic acid (FIG. 4). ACMS can also be converted to picolinic acid, a metabolite that cannot be converted into $\mathrm{NAD}^{+}$, by ACMS decarboxylase (ACMSD). Thus, ACMSD reduces flux from tryptophan to $\mathrm{NAD}^{+}$. In Caenorhabditis elegans, complete inhibition of the ACMSD orthologue boosted NAD ${ }^{+}$levels and extended lifespan ${ }^{54}$. ACMSD inhibitors also ameliorated the pathological phenotypes in a mouse model of dietinduced fatty liver disease and in two mouse models of AKI (renal IRI and cisplatin).

An enzyme that acts upstream of ACMSD in the de novo pathway, kynurenine-3-monooxygenase (KMO), converts kynurenine into its 3-hydroxylated form (3OH-kynurenine). KMO competes with kynurenine aminotransferases (KATs) that generate kynurenic acid. A 2019 study reported that global Kmo-knockout mice were less susceptible to renal IRI than wild-type controls ${ }^{55}$. This finding suggests an adaptive role of the suppression of de novo pathway enzymes, namely, the shunting of metabolic flux away from $\mathrm{NAD}^{+}$biosynthesis and towards the generation of kynurenic acid, which modulates inflammation, neuronal signalling and adaptive immunity ${ }^{56}$. However, this study reported that Kmo-knockout mice did not develop spontaneous proteinuria, a finding contradicted by an earlier independent report on Kmo-knockout mice showing that they did develop spontaneous proteinuria ${ }^{57}$. Therefore, further study of KMO is needed.

Consequences of reduced $\mathrm{NAD}^{+}$levels. As described above, a combination of accelerated $\mathrm{NAD}^{+}$consumption and diminished $\mathrm{NAD}^{+}$biosynthesis appears to underlie the fall in renal $\mathrm{NAD}^{+}$levels that occurs following AKI. The mammalian renal cortex relies heavily on fatty acid oxidation (FAO) to generate the ATP that is necessary for solute transport ${ }^{58}$. Aligning with the concept that $\mathrm{NAD}^{+}$deficiency in $\mathrm{AKI}$ results in impaired FAO, several studies have reported intratubular accumulation of fats following various forms of experimental $\mathrm{AKI}^{58-61}$. Together, these results suggest that the low levels of renal tubular ATP that are associated with AKI could result from an $\mathrm{NAD}^{+}$-dependent defect in $\mathrm{FAO}$, which also results in intracellular lipid accumulation ${ }^{60,61}$.

A reduction in FAO could contribute to AKI by reducing the level of ATP that is available for essential tubular functions and/or via the toxic effects of accumulated lipids. Compared with wild-type littermates, we found that mice with renal PGC1a overexpression had higher levels of the FAO product $\beta$-hydroxybutyrate, which in turn signalled increased production of prostaglandin $\mathrm{E}_{2}\left(\mathrm{PGE}_{2}\right)$, a known renal vasodilator ${ }^{62}$. This finding suggests that substrates of fuel oxidation in the epithelial compartment enable the vasculature to regulate blood flow in order to maintain adequate oxygen delivery. Lipidomic analysis showed that 
AKI-induced lipid accumulation is characterized by a striking enrichment of diacylglycerols and triacylglycerols high in polyunsaturated fatty acid (PUFA) content. The $>50-100$-fold elevations of these PUFArich lipid species are greatly out of proportion compared with total lipid accumulation, suggesting that, in addition to blocking their consumption via FAO, AKI might also increase their synthesis. A subsequent study showed that an increase in lipid desaturation may be an adaptive response to the reduced $\mathrm{NAD}^{+}: \mathrm{NADH}$ ratio in $\mathrm{AKI}$, providing a mechanism for $\mathrm{NAD}^{+}$recycling when tubular mitochondrial respiration is impaired (FIG. 5). For example, the addition of a desaturase inhibitor further lowered the $\mathrm{NAD}^{+}: \mathrm{NADH}$ ratio and worsened AKI in mice treated with a mitochondrial toxin ${ }^{63}$. In our study, administration of Nam not only fortified renal $\mathrm{NAD}^{+}$but also reduced AKI-associated accumulation of fats (including PUFA-rich diacylglycerols and triacylglycerols) and AKI severity in two distinct models: IRI and cisplatin nephrotoxicity ${ }^{47}$. These findings suggest that reduced $\mathrm{NAD}^{+}$levels in various types of renal injury might result in impairment of normal tubular function, but might be amenable to therapeutic augmentation.

Additional studies support a critical role for $\mathrm{NAD}^{+}$ biosynthesis in counteracting the effects of diverse renal stressors. Administration of the $\mathrm{NAD}^{+}$precursor $\mathrm{NMN}$, ameliorated IRI and cisplatin nephrotoxicity in aged mice $^{64}$. This study found that the beneficial effects of

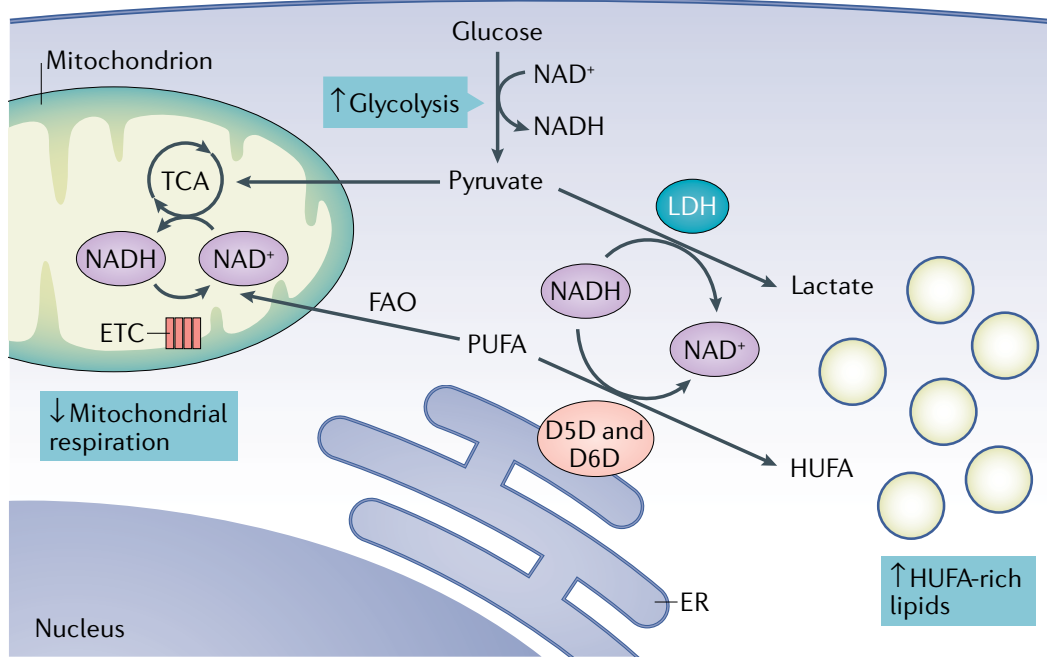

Fig. 5 | Glycolytic NAD+ recycling and lipid accumulation in AKI. Nicotinamide adenine dinucleotide $\left(\mathrm{NAD}^{+}\right)$is reduced to $\mathrm{NADH}$ via glycolysis in the cytosol and via the tricarboxylic acid (TCA) cycle and $\beta$-oxidation of fatty acids (FAO) in mitochondria. Under aerobic conditions, $\mathrm{NAD}^{+}$is regenerated as electrons are transported to the mitochondrial electron transport chain (ETC) to drive oxidative phosphorylation, which produces ATP. During acute kidney injury (AKI), mitochondrial respiration and function are impaired, glycolysis increases to meet cellular energy demands and cytosolic NAD recycling is required. Lactate dehydrogenase $(\mathrm{LDH})$, which converts pyruvate to lactate, has a major role in cytosolic $\mathrm{NAD}^{+}$recycling. Desaturation of polyunsaturated fatty acids (PUFAs) to form highly unsaturated fatty acids (HUFAs) is an additional mechanism of glycolytic NAD ${ }^{+}$recycling that is mediated by delta- 5 and delta- 6 fatty acid desaturases (D5D and D6D). D5D and D6D are located in the endoplasmic reticulum (ER) membrane and have cytosol-facing catalytic domains; they are highly expressed in the kidney and liver. Experimental AKI is associated with $>50-100$-fold increases in the levels of HUFA-containing triglycerides and cellular lipid accumulation in renal tubular cells.
NMN were attenuated in mice with SIRT1 deficiency. We found that treatment with NMN preserved mitophagy and enhanced lysosomal disposal of injured mitochondria in cisplatin-treated tubular cells and in a mouse model of cisplatin nephrotoxicity ${ }^{49}$. PGC1 $\alpha$ and $\mathrm{NAD}^{+}$are upstream inducers of transcription factor $\mathrm{EB}$, a master regulator of lysosomal biogenesis ${ }^{65,66}$. Administration of $\mathrm{NAD}^{+}$precursors (Nam or NMN) may therefore not only enable efficient ATP generation via FAO but also promote mitochondrial quality control by coordinating the safe disposal of injured, pro-oxidative mitochondria in lysosomes. Indeed, earlier work showed that another cytoprotective sirtuin enzyme, SIRT3, utilizes $\mathrm{NAD}^{+}$to prevent mitochondrial fragmentation and protect renal function in the setting of cisplatin exposure ${ }^{67}$. An independent study confirmed a beneficial effect of SIRT3 in a model of septic AKI ${ }^{68}$.

Finally, a study of nitric oxide-dependent posttranslational protein modifications in the renal tubule implicated $\mathrm{NADP}^{+}$imbalance in the pathogenesis of renal IRI ${ }^{69}$. Whether $\mathrm{NAD}^{+}$augmentation achieves renal benefit directly by enhancing FAO or indirectly via one or several cell-regulatory networks, the enhancement of $\mathrm{NADP}^{+}$levels or a combination of these mechanisms remains to be elucidated (FIG. 6). As $\mathrm{NAD}^{+}$and $\mathrm{NADP}^{+}$ participate in several hundred redox reactions and $>20$ enzymatic reactions involve consumption of $\mathrm{NAD}^{+}$ $\left(\mathrm{REF}^{70}\right)$, it may be difficult and perhaps overly simplistic to implicate a single downstream effector pathway in AKI. Instead, AKI can be considered a transient state of impaired $\mathrm{NAD}^{+}$homeostasis that results in multiple deleterious consequences for injured cells.

Dysregulation of $\mathrm{NAD}^{+}$homeostasis in human AKI. In line with findings in animal models, people at risk of AKI seem to exhibit perturbations in mitochondrial metabolism and $\mathrm{NAD}^{+}$homeostasis. We showed that the renal expression of PGC1 $a$ is suppressed in biopsy samples from patients with AKI compared with the levels in nephrectomy controls ${ }^{47}$. Our subsequent pilot study examined the relationship between PGC1 $\alpha$ expression in renal allografts from deceased donors and delayed graft function (DGF) ${ }^{71}$. In deceased-donor kidney transplants, periods of warm and cold ischaemia prior to implantation are thought to contribute to clinical IRI. Although the majority of deceased-donor grafts produce sufficient renal clearance for the recipient to cease dialysis within the first $48 \mathrm{~h}$ post-transplantation, recipients with DGF may require dialysis for several weeks. In this setting, allograft biopsies are often performed postoperatively to rule out acute rejection. In a small cohort of decreased-donor transplant recipients who had DGF without acute rejection, we found that allograft biopsy samples obtained within 30 days post-transplantation exhibited a large spread of PGC1 $\alpha$ expression in morphologically intact tubules. Biopsy samples that had the lowest PGC1 $\alpha$ expression were associated with an additional 2 weeks of dialysis compared with those with the highest PGCla expression ${ }^{71}$. Although preliminary, this result suggests a quantitative relationship between mitochondrial metabolism and clinically relevant end points in AKI. 


\author{
Outbred mice \\ In contrast to most laboratory \\ strains, which are inbred, \\ outbred mice maintain genetic \\ diversity and phenotypic \\ variation. Outbred mice are \\ therefore useful for identifying \\ genetic factors linked to \\ phenotypes of interest.
}

The finding that reduced QPRT expression increased AKI susceptibility in mice led us to assess whether changes in urinary quinolinic acid levels were associated with risk of clinical $\mathrm{AKI}^{1}$. We measured the levels of quinolinic acid, tryptophan and additional de novo

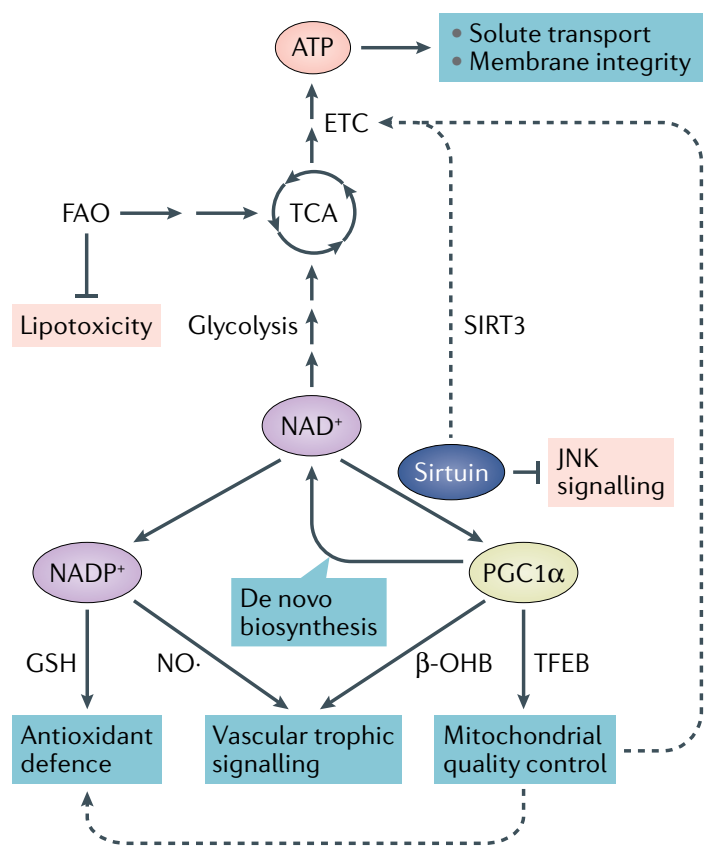

Fig. 6 | Potential renoprotective actions of NAD ${ }^{+}$. Nicotinamide adenine dinucleotide $\left(\mathrm{NAD}^{+}\right)$is required for distinct reactions in glycolysis, fatty acid oxidation (FAO) and the tricarboxylic acid (TCA) cycle that lead to the generation of ATP via the electron transport chain (ETC) in mitochondria. In healthy renal tubular epithelial cells, this ATP is utilized to provide the energy required for key functions, such as solute transport and maintenance of membrane integrity. Efficient FAO also prevents the potentially toxic accumulation of storage fats. $\mathrm{NAD}^{+}$might also exert renoprotective effects via its interactions with sirtuins and PPAR $\gamma$ co-activator $1 \alpha$ (PGC1 $1 \alpha)$. Sirtuin 1 (SIRT1) activates PGC1 $\alpha$ via $\mathrm{NAD}^{+}$-dependent deacetylation and PGC1 $a$ in turn promotes $\mathrm{NAD}^{+}$biosynthesis via the de novo pathway by co-ordinately upregulating the expression of the genes encoding enzymes in this pathway. PGC1 $1 \alpha$ promotes mitochondrial quality control and ATP production via interrelated process that include mitochondrial biogenesis and the induction of mitophagy via transcription factor EB (TFEB). SIRT3 also utilizes NAD to directly promote healthy mitochondrial function and SIRT1 may limit stress signalling through the pro-apoptotic Jun $\mathrm{N}$-terminal kinase (JNK) pathway. In addition to its effects on mitochondria, PGC1 1 signalling can induce the production of vascular trophic molecules, such as VEGF. In tubular epithelial cells, a PGC1 1 -dependent product of FAO, $\beta$-hydroxybutyrate ( $\beta-\mathrm{OHB})$, may signal the production of vasodilator prostaglandins that can maintain renal blood flow during conditions such as shock that would otherwise promote renal ischaemia. Phosphorylation of $\mathrm{NAD}^{+}$to $\mathrm{NADP}^{+}$may potentiate defence against oxidant stress induced by inflammation, toxins, or ischaemia-reperfusion injury by promoting the reduction of glutathione (GSH) and through the vasodilator nitric oxide (NO•). Mitochondrial quality control may also limit the burden of free radicals emanating from injured mitochondria. pathway metabolites in urine samples obtained from 215 critically ill patients within the first $24 \mathrm{~h}$ of intensive care unit admission. In this prospectively enrolled cohort, 51 patients developed AKI. The median time from urine sample collection to AKI diagnosis was 2.5 days. We found that increased urinary quinolinic acid levels and increased urinary quinolinic acid:tryptophan ratio were quantitatively and independently associated with increased risk of AKI, need for renal replacement therapy and related adverse outcomes. These clinical findings mirror the results in mice, suggesting that experimental animals and patients develop a dynamic block in de novo $\mathrm{NAD}^{+}$biosynthesis when exposed to AKI-inducing stressors.

Finally, to test the preclinical finding that exogenous Nam can ameliorate AKI when QPRT levels are suppressed, we designed a small, placebo-controlled, blinded and randomized trial of peri-operative oral Nam ( $1 \mathrm{~g}$ or $3 \mathrm{~g}$ daily) in patients undergoing on-pump cardiac surgery, which causes renal ischaemia ${ }^{1}$. There was no difference between either dose of Nam and placebo in terms of safety. The pharmacokinetics of Nam were consistent with a water-soluble vitamin, demonstrating clearance in the urine, and evidence of conversion of Nam to $\mathrm{NAD}^{+}$was ascertained. Finally, serial measurements of serum creatinine levels suggested a potential renal benefit of Nam administration. As only 41 patients completed the trial, these encouraging results require further testing in larger, multicentre studies.

In summary, complementary systems biology approaches across multiple genetically modified mice and several AKI models have identified dynamic defects in renal $\mathrm{NAD}^{+}$homeostasis that may be measurable in humans and targetable using simple vitamin therapy.

\section{Chronic kidney disease}

Partial recovery of AKI or repeated episodes of AKI can lead to CKD. Conversely, CKD is a major risk factor for AKI. These clinical relationships suggest a mechanistic connection between AKI and CKD. An intriguing body of work in experimental models of CKD and clinical observational studies suggest tantalizing links to $\mathrm{NAD}^{+}$ metabolism.

Mice with homozygous knockout of a bidirectional promoter that regulates the genes encoding the $\mathrm{NAD}^{+}$ de novo biosynthesis pathway enzyme arylformamidase and the enzyme thymidine kinase had shortened lifespans, spontaneous glomerulosclerosis, kidney failure and elevations in the circulating levels of upstream metabolites including kynurenine $\mathrm{e}^{72,73}$. These findings are difficult to interpret because the knockout also affected thymidine kinase, which is required for nucleotide synthesis for DNA replication and thus expressed in virtually all cells, but is not known to affect NAD ${ }^{+}$ homeostasis directly.

A study that used genome-wide association mapping in 290 outbred mice to identify genetic variants linked to urinary albumin:creatinine ratios found a highly significant variant tagging an interval in chromosome 1 that includes the $K M O$ gene $^{74}$. A follow-up study found that glomerular expression of $\mathrm{KMO}$ was suppressed in mice and humans with diabetes compared with normal 
controls $^{57}$. Furthermore, knockdown of the KMO orthologue in zebrafish and knockout of Kmo in mice led to a spontaneous proteinuria phenotype ${ }^{57}$. Although renal KMO expression was more abundant in the tubules than in the glomeruli, the renal histological abnormalities were confined to the glomerulus in both model organisms. The zebrafish KMO knockdown showed pronounced podocyte foot process effacement, whereas the mouse Kmo knockout showed mild foot process effacement. As podocytes are affected in many CKD syndromes, these results suggest an involvement of de novo $\mathrm{NAD}^{+}$biosynthesis in CKD. Spontaneous proteinuria was not, however, observed in an independently generated $\mathrm{Kmo}$-knockout mouse model ${ }^{55}$. These results suggest the need to investigate the contribution of KMO to podocyte function more closely, perhaps with evaluation of podocyte versus tubular-specific knockout mice.

A study that more directly addressed a contribution of $\mathrm{NAD}^{+}$metabolism in CKD identified protective effects of exogenous Nam in the murine unilateral ureteral obstruction (UUO) model of renal fibrosis ${ }^{75}$. Nam treatment reduced fibrosis, tubular atrophy, apoptosis, immune cell infiltration and renal cytokine expression following UUO. In addition, Nam blocked the transforming growth factor- $\beta$ (TGF $\beta$ )-induced expression of fibrotic proteins in cultured mouse proximal tubular cells. Experiments to induce renal fibrosis in genetic mouse models targeting enzymes involved in $\mathrm{NAD}^{+}$ homeostasis should help to illuminate the extent to which intact tubular cell $\mathrm{NAD}^{+}$defends against tubular atrophy and interstitial scarring.

A large body of research suggests that dysregulation of $\mathrm{NAD}^{+}$metabolism might contribute to obesity, metabolic syndrome, diabetes and diabetic end-organ complications $^{36}$. The pathogenesis of diabetic kidney disease has also been linked to $\mathrm{NAD}^{+}$metabolism. In mice, streptozotocin (STZ) treatment (a model of diabetes) resulted in lower renal tubular levels of the $\mathrm{NAD}^{+}$dependent enzyme SIRT1 $\left(\mathrm{REF}^{76}{ }^{76}\right.$. Moreover, proximal tubule-specific Sirt1 overexpression prevented and Sirt1 knockout exacerbated glomerular changes in this model of diabetes. Furthermore, culturing proximal tubular cells in high glucose resulted in lower levels of NMN and $\mathrm{NAD}^{+}$relative to normal glucose conditions. Another study reported that NMN attenuates renal fibrosis in the STZ-treated rat model by inhibiting Nam phosphoribosyltransferase (NAMPT), which is the major biosynthetic enzyme in the salvage pathway ${ }^{77}$. This unexpected mechanism requires further investigation.

Additional studies in models of CKD have implicated factors that are relevant to $\mathrm{NAD}^{+}$homeostasis, particularly PGC1 1 , without directly assessing $\mathrm{NAD}^{+}$levels. For example, PGC1 a expression is reduced in the kidneys of mice with STZ-induced diabetes ${ }^{78}$. In this model, treatment with an AMPK activator increased PGC1 $\alpha$ expression, reduced the levels of glomerular fibronectin and TGF $\beta$ and reduced albuminuria. The long non-coding RNA Tug1 may also modulate PGC1a expression in experimental diabetes. Podocyte-specific overexpression of Tug1 increased the expression of PGC1 $\alpha$ and its transcriptional targets and improved the histological features of diabetic nephropathy in a $d b / d b$ model $^{79}$.
However, the results of another study suggest that supranormal PGC1a induction in the podocyte could promote a collapsing glomerulopathy marked by severe proteinuria and progressive renal failure ${ }^{80}$. However, overexpression of PGC1 $\alpha$ in the podocyte, a cell type that normally expresses very little PGC1 1 relative to the cortical renal tubular epithelium ${ }^{50}$, seems to have fundamentally altered its normal fuel metabolism, shifting it from normal glucose consumption towards $\mathrm{FAO}^{80}$. As a result, determining the physiological contributions of podocyte PGC1a to renal health and disease will require further study.

A transcriptomic analysis found that microdissected tubulointerstitial samples from patients with diabetic or hypertensive CKD had significantly lower expression of PGCla and downstream genes involved in FAO than those from people with normal kidney function ${ }^{81}$. Echoing findings in experimental $\mathrm{AKI}^{60,61}$, lower expression of these genes was associated with higher lipid accumulation in the tubular epithelium. The researchers also used folic acid to induce tubulointerstitial fibrosis in mice and found that transgenic expression of PGCla in the renal tubular epithelium restored the expression of FAO genes, reduced expression of profibrotic molecules and improved renal histology. A related study from the same group showed that distal tubular deletion of the gene encoding LKB1, an upstream kinase that activates AMPK, was associated with decreased expression of AMPK and PGC1 $\alpha$ and resulted in progressive kidney disease in mice ${ }^{82}$. LKB1-deficient cells were characterized by energy depletion, reduced FAO and a profibrotic phenotype; treatment with an AMPK agonist ameliorated these effects. Taken together, these results raise the possibility that metabolic dysfunction might be a common characteristic that links AKI and CKD. Whether increasing PGC1 $\alpha$ expression either directly or via AMPK or Tug1 in CKD models is associated with changes in kidney $\mathrm{NAD}^{+}$levels has not yet been reported. However, the encouraging result with exogenous Nam administration in a mouse model of fibrosis suggests that augmentation of $\mathrm{NAD}^{+}$is a potential therapeutic approach to slowing CKD progression ${ }^{75}$.

Altered tryptophan metabolism has been identified in several metabolomics studies of patients with CKD. For example, lower baseline levels of tryptophan and higher levels of downstream metabolites (including quinolinic acid) were associated with the development of incident CKD in several large cohorts, including cohorts from the Framingham Heart Study ${ }^{83}$, Cooperative Health Research in the Augsburg Region study ${ }^{84}$ and Atherosclerosis Risk in Communities study ${ }^{85}$. Whether these findings reflect perturbations in de novo $\mathrm{NAD}^{+}$ biosynthesis and, if so, whether these perturbations are modifiable remains unknown.

In a randomized trial of oral Nam in patients with CKD from 2019 (REF. ${ }^{86}$ ), 205 participants with a mean estimated glomerular filtration rate (eGFR) of $32 \mathrm{ml} / \mathrm{min} / 1.73 \mathrm{~m}^{2}$ received either Nam (750 mg twice daily), lanthanum carbonate, both Nam and lanthanum carbonate or placebo for 12 months. Nam was administered in this study because it inhibits active intestinal phosphate transport and the primary end points were 
Box 1 | Data linking NAD ${ }^{+}$homeostasis to renal health

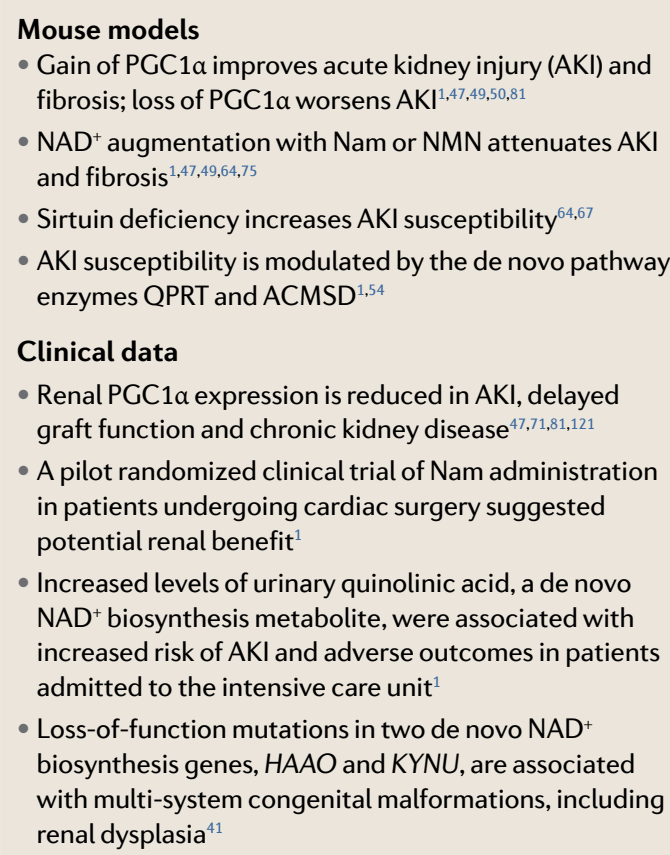

changes in serum phosphate and fibroblast growth factor 23 levels from baseline. No significant differences in these mineral metabolism parameters or in mean change in eGFR from baseline at 12-month follow-up were observed between the study groups. Although these findings suggest that boosting $\mathrm{NAD}^{+}$with Nam might not be beneficial in CKD, this study did not assess whether Nam administration modified $\mathrm{NAD}^{+}$ homeostasis (for example, by analysing blood or urine metabolites). Furthermore, the study size and duration of follow-up were modest, and the rate of drug discontinuation was high ( $42 \%$ in the Nam plus lanthanum group and $25 \%$ in the Nam only group). Gastrointestinal symptoms and pill burden were the most common reasons cited for discontinuation. Thus, the tolerability of higher doses of Nam should be carefully considered when designing future studies.

\section{Age-related loss of kidney function}

A decline in $\mathrm{NAD}^{+}$levels is closely linked to the metabolic changes and reduction in mitochondrial function that accompanies ageing. This relationship was first demonstrated in yeast in which both Sir2 and maintenance of $\mathrm{NAD}^{+}$levels were shown to be essential for the increase in longevity that is associated with caloric restriction ${ }^{87}$. In mice, levels of $\mathrm{NAD}^{+}$in skeletal muscle have been shown to decrease with increasing age ${ }^{88}$. Tissue levels of $\mathrm{NAD}^{+}$also decline with age in the kidney, heart, lung and liver of rats ${ }^{89}$. As several proteins with crucial roles in regulating cell metabolism, including SIRT1, are dependent on $\mathrm{NAD}^{+}$as a substrate, a decline in $\mathrm{NAD}^{+}$levels may be a determining factor that leads to impairment of mitochondrial function with age $^{88}$. Evidence for a decline in $\mathrm{NAD}^{+}$levels with age in humans is limited. However, a study that used in vivo high-field magnetic resonance imaging reported that the levels of $\mathrm{NAD}^{+}$and the $\mathrm{NAD}^{+}: \mathrm{NADH}$ ratio in the healthy human brain decline with age ${ }^{90}$.

A decline in renal $\mathrm{NAD}^{+}$levels may be partially responsible for the increase in incidence of AKI with advancing age $^{91}$. Age $>65$ years has been associated with nonrecovery from AKI and progression to moderate or severe CKD following $\mathrm{AKI}^{92-94}$. Kidney function declines with ageing, even among healthy populations ${ }^{95-97}$, and whether this age-related loss of kidney function should be classified as CKD - as is currently the case - remains a subject of debate ${ }^{98}$. $\mathrm{NAD}^{+}$augmentation is an attractive therapeutic strategy to mitigate the adverse effects of ageing on cellular metabolism ${ }^{88}$. Although little is known about the relationship between declining $\mathrm{NAD}^{+}$levels and $\mathrm{CKD}$, it is plausible that augmentation of $\mathrm{NAD}^{+}$may also have a therapeutic role in slowing CKD progression. More work is needed to develop methods that can interrogate $\mathrm{NAD}^{+}, \mathrm{NADH}, \mathrm{NADP}^{+}, \mathrm{NADPH}$, their respective precursors and their intermediates in kidney tissue to understand how $\mathrm{NAD}^{+}$metabolism is impacted by ageing, both in experimental organisms and in humans ${ }^{99}$.

\section{Future perspectives}

$\mathrm{NAD}^{+}$metabolism is intimately involved in the core function of the nephron: the transport of solutes against powerful chemical gradients ${ }^{100,101}$. Studies in the past 3 years have illuminated new aspects of the mechanisms that lead to deficiency of $\mathrm{NAD}^{+}$metabolism following acute and chronic stressors and the physiological consequences of this deficiency (BOX 1). However, several important questions need to be addressed. First, a highresolution map of the anatomical regulation within the kidney of enzymatic pathways for $\mathrm{NAD}^{+}$biosynthesis, $\mathrm{NAD}^{+}$consumption and the transport of precursors and intermediates is needed to aid functional understanding of $\mathrm{NAD}^{+}$metabolism in this highly heterogeneous organ. For example, a sodium-linked cotransporter, SLC12A8, has been identified as the molecule responsible for NMN uptake in the gut ${ }^{4}$, but whether this transporter also aids $\mathrm{NAD}^{+}$precursor reabsorption from the renal filtrate is unknown.

Second, whether aspects of kidney protection downstream of $\mathrm{NAD}^{+}$metabolism are attributable to particular effectors remains unknown. For example, whether the anabolic reactions that are catalysed by NADPH are more or less important than the oxidative reactions that are catalysed by $\mathrm{NAD}^{+}$in the setting of acute stress remains to be determined. Greater understanding of these nuances could aid the development of more highly targeted treatments.

Third, the encouraging findings of the randomized clinical trial of high-dose oral Nam in perioperative AKI discussed above suggest that dietary precursors of $\mathrm{NAD}^{+}$could potentially be used to increase renal resilience $^{1}$. An inexpensive and safe vitamin-based intervention for renoprotection is an attractive prospect. Oral $\mathrm{NR}$ and NMN are converted to Nam in the liver before transformation to $\mathrm{NAD}^{+}$, suggesting that oral Nam might be the most efficient dietary $\mathrm{NAD}^{+}$precursor ${ }^{8}$. Pharmacological treatments are also being developed to boost $\mathrm{NAD}^{+}$metabolism by stimulating NAMPT ${ }^{102}$, inhibiting $\mathrm{ACMSD}^{54}$ and inhibiting $\mathrm{NAD}^{+}$-consuming 
Ataxia telangiectasia

A rare inherited disorder that leads to diminished motor coordination (ataxia) and the development of reddish lesions on the skin and mucous membranes (telangiectasia). enzymes $^{21,103}$. We therefore anticipate a substantial increase in clinical trials to test nutritional and pharmacological interventions, either singly or in combination, across a broad swathe of medical indications.

Given the ubiquity and importance of $\mathrm{NAD}^{+}$metabolism in cellular health, knowledge gained in the kidney may provide novel fundamental and translational insights that could be applicable to other organs. Similar to the kidney tubular epithelium, cardiomyocytes are mitochondria-rich and highly dependent on FAO for ATP generation. Enhancing cardiac NAD ${ }^{+}$levels by transgenic expression of NAMPT or exogenous administration of NMN seems to prevent the decrease in $\mathrm{NAD}^{+}$and attenuate the cardiac damage that results from IRI ${ }^{104,105}$. Although neurons do not utilize fatty acids for ATP generation, they are metabolically active and cerebral ischaemia results in $\mathrm{NAD}^{+}$depletion in the central nervous system ${ }^{106}$. In mice, treatment with Nam or NMN normalized brain $\mathrm{NAD}^{+}$levels and ameliorated brain damage following cerebral ischaemia ${ }^{107}$. Low $\mathrm{NAD}^{+}$levels and mitochondrial dysfunction have also been observed in animal models of ataxia telangiectasia; replenishing $\mathrm{NAD}^{+}$via $\mathrm{NR}$ administration normalized neuromuscular function and delayed memory loss in these models ${ }^{108}$. In the liver, $\mathrm{NAD}^{+}$augmentation through various pathways has been shown to protect against diet-induced obesity and fatty-liver disease ${ }^{54,109}$. Thus, $\mathrm{NAD}^{+}$seems to have a key role in maintaining health in multiple metabolically active organs. As different tissues vary in their energy demands, preferred fuels, dependence on de novo $\mathrm{NAD}^{+}$biosynthesis versus $\mathrm{NAD}^{+}$salvage and expression of downstream $\mathrm{NAD}^{+}$-consuming enzymes, a more nuanced understanding of $\mathrm{NAD}^{+}$homeostasis throughout the body is required to harness its full potential for therapeutic modulation (TABLE 1).

\section{Conclusions}

The regulation of $\mathrm{NAD}^{+}$balance within cells involves a complex interplay among biosynthetic pathways and degradative enzymes, compartmentalization and movement between pools of $\mathrm{NAD}(\mathrm{H})$ and $\mathrm{NADP}(\mathrm{H})$. A body of research dating back nearly 20 years has identified $\mathrm{NAD}^{+}$ as a determinant of ageing in experimental organisms; however, translating knowledge of $\mathrm{NAD}^{+}$biology into medical applications is challenging. As a highly metabolically active organ with a clear age-related phenotype, the
Table 1 | NAD ${ }^{+}$-based therapies: potential indications

\begin{tabular}{|llr|}
\hline Organ & Disease & Refs \\
\hline Kidney & Acute kidney injury & $1,47,54,64$ \\
\cline { 2 - 3 } Heart & Chronic kidney disease & 75 \\
& Heart failure & 111 \\
\cline { 2 - 3 } Skeletal muscle & Ischaemia-reperfusion injury & 1,104 \\
\cline { 2 - 3 } & Muscular dystrophies & 112 \\
\cline { 2 - 3 } Liver & Sarcopenia & 113 \\
& Metabolic syndrome & 109,114 \\
\cline { 2 - 3 } Nervous system & Fatty liver disease & 54 \\
& Alzheimer disease & 115 \\
& Parkinson disease & 116 \\
\cline { 2 - 3 } & Ataxia telangiectasia & 117 \\
\hline Eye & Peripheral axonopathies & 103,118 \\
\hline Skin & Glaucoma & 119 \\
\hline & Cancer prevention & 120 \\
\hline
\end{tabular}

kidney has emerged as an intriguing physiological system in which to study the consequences of $\mathrm{NAD}^{+}$biology. In tubular epithelial cells, $\mathrm{NAD}^{+}$facilitates the high demand for electron transport that transforms fatty acid fuel into ATP. Experimental AKI of various aetiologies is characterized by a common, rapid decrease in renal $\mathrm{NAD}^{+}$that is driven in part by impaired biosynthesis from tryptophan. Augmentation of $\mathrm{NAD}^{+}$enhances FAO and broad cell-regulatory signalling networks that protect oxidative metabolism and mitochondrial health. Together, these actions may protect stressed renal tubular cells from death. Human AKI also seems to be characterized by a deficit in de novo $\mathrm{NAD}^{+}$biosynthesis, raising the possibility of therapeutic $\mathrm{NAD}^{+}$augmentation using vitamin $\mathrm{B}_{3}$ analogues. Future studies are needed to understand the mechanistic links among ageing, CKD and AKI. Study of $\mathrm{NAD}^{+}$in the kidney may also yield insights that are applicable to other metabolically active organs, such as the heart and brain. In addition, translational studies that assess and modulate $\mathrm{NAD}^{+}$metabolism may lead to new avenues for the diagnosis, prognosis, prevention and treatment of diseases of the kidney and other organs.

Published online 31 October 2019
1. Poyan Mehr, A. et al. De novo NAD biosynthetic impairment in acute kidney injury in humans. Nat. Med. 24, 1351-1359 (2018).

2. Evans, J., Wang, T. C., Heyes, M. P. \& Markey, S. P. $\mathrm{LC} / \mathrm{MS}$ analysis of NAD biosynthesis using stable isotope pyridine precursors. Anal. Biochem. 306 197-203 (2002)

3. Revollo, J. R., Grimm, A. A. \& Imai, S. The NAD biosynthesis pathway mediated by nicotinamide phosphoribosyltransferase regulates Sir2 activity in mammalian cells. J. Biol Chem 279, 50754-50763 (2004).

4. Grozio, A. et al. Slc12a8 is a nicotinamide mononucleotide transporter. Nat. Metab. 1, 47-57 (2019).

5. Carpenter, K. J. The relationship of pellagra to corn and the low availability of niacin in cereals. Experientia Suppl. 44, 197-222 (1983).

6. Krehl, W. A., Teply, L. J., Sarma, P. S. \& Elvehjem, C. A. Growth-retarding effect of corn in nicotinic acid-low rations and its counteraction by tryptophane. Science 101, 489-490 (1945)

7. Collins, P. B. \& Chaykin, S. The management of nicotinamide and nicotinic acid in the mouse. J. Biol. Chem. 247, 778-783 (1972).

8. Liu, L. et al. Quantitative analysis of NAD synthesisbreakdown fluxes. Cell Metab. 27, 1067-1080.e5 (2018).

9. Trammell, S. A. et al. Nicotinamide riboside is uniquely and orally bioavailable in mice and humans. Nat Commun 7,12948 (2016).

10. Martens, C. R. et al. Chronic nicotinamide riboside supplementation is well-tolerated and elevates NAD in healthy middle-aged and older adults. Nat. Commun 9, 1286 (2018).

11. Hanson, J. et al. Nicotinic acid- and monomethyl fumarate-induced flushing involves GPR109A expressed by keratinocytes and COX-2-dependent prostanoid formation in mice. J. Clin. Invest. 120, 2910-2919 (2010).
12. Michan, S. \& Sinclair, D. Sirtuins in mammals: insights into their biological function. Biochem. J. 404, 1-13 (2007).

13. Bonkowski, M. S. $\propto$ Sinclair, D. A. Slowing ageing by design: the rise of $\mathrm{NAD}^{+}$and sirtuin-activating compounds. Nat. Rev. Mol. Cell Biol. 17, 679-690 (2016).

14. Kim, S., Benguria, A., Lai, C. Y. \& Jazwinski, S. M. Modulation of life-span by histone deacetylase genes in Saccharomyces cerevisiae. Mol. Biol. Cell 10, 3125-3136 (1999).

15. Kaeberlein, M., McVey, M. \& Guarente, L. The SIR2/3/4 complex and SIR2 alone promote longevity in Saccharomyces cerevisiae by two different mechanisms. Genes Dev. 13, 2570-2580 (1999).

16. Imai, S., Armstrong, C. M., Kaeberlein, M. $\&$ Guarente, L. Transcriptional silencing and longevity protein Sir2 is an NAD-dependent histone deacetylase. Nature 403, 795-800 (2000). 
17. Guarente, L. Sirtuins in aging and disease. Cold Spring Harb. Symp. Quant. Biol. 72, 483-488 (2007).

18. Dang, W. The controversial world of sirtuins Drug Discov. Today Technol. 12, e9-e17 (2014).

19. Camacho-Pereira, J. et al. CD38 dictates age-related NAD decline and mitochondrial dysfunction through an SIRT3-dependent mechanism. Cell Metab. 23 1127-1139 (2016)

20. Aksoy, P., White, T. A., Thompson, M. \& Chini, E. N. Regulation of intracellular levels of NAD: a novel role for CD38. Biochem. Biophys. Res. Commun. 345 1386-1392 (2006)

21. Tarrago, M. G. et al. A potent and specific CD38 inhibitor ameliorates age-related metabolic dysfunction by reversing tissue $\mathrm{NAD}^{+}$decline Cell Metab. 27, 1081-1095.e10 (2018).

22. Rechsteiner, M., Hillyard, D. \& Olivera, B. M. Turnover at nicotinamide adenine dinucleotide in cultures of human cells. J. Cell. Physiol. 88, 207-217 (1976)

23. Cambronne, X. A. et al. Biosensor reveals multiple sources for mitochondrial $\mathrm{NAD}^{+}$. Science 352. 1474-1477 (2016).

24. Williams, G. T., Lau, K. M., Coote, J. M. \& Johnstone, A. P. NAD metabolism and mitogen stimulation of human lymphocytes. Exp. Cell Res. 160, 419-426 (1985).

25. Bai, P et al. PARP-1 inhibition increases mitochondria metabolism through SIRT 1 activation. Cell Metab. 13 461-468 (2011)

26. Wamelink, M., Struys, E. \& Jakobs, C. The biochemistry, metabolism and inherited defects of the pentose phosphate pathway: a review. J. Inherit. Metab. 31 703-717 (2008)

27. Soderberg, T. Biosynthesis of ribose-5-phosphate and erythrose-4-phosphate in archaea: a phylogenetic analysis of archaeal genomes. Archaea 1, 347-352 (2005).

28. Kawai, S. \& Murata, K. Structure and function of NAD kinase and NADP phosphatase: key enzymes that regulate the intracellular balance of $N A D(H)$ and NADP(H). Biosci. Biotechnol. Biochem. 72, 919-930 (2008).

29. Leopold, J. A. et al. Aldosterone impairs vascular reactivity by decreasing glucose-6-phosphate dehydrogenase activity. Nat. Med. 13, 189-197 (2007).

30. Ratliff, B. B., Abdulmahdi, W., Pawar, R. \& Wolin, M. S Oxidant mechanisms in renal injury and disease. Antioxid. Redox Signal. 25, 119-146 (2016).

31. Ruderman, N. B. et al. AMPK and SIRT 1: a long standing partnership? Am. J. Physiol. Endocrinol. Metab. 298, E751-E760 (2010)

32. Canto, C. \& Auwerx, J. PGC- $1 \alpha$, SIRT 1 and AMPK an energy sensing network that controls energy expenditure. Curr. Opin. Lipidol. 20, 98-105 (2009).

33. Mouchiroud, L. et al. The $\mathrm{NAD}^{+} /$sirtuin pathway modulates longevity through activation of mitochondrial UPR and FOXO signaling. Cell 154 430-441 (2013).

34. Jovaisaite, V. \& Auwerx, J. The mitochondrial unfolded protein response-synchronizing genomes. Curr. Opin. Cell Biol. 33, 74-81 (2015).

35. Bai, P. et al. PARP-2 regulates SIRT1 expression and whole-body energy expenditure. Cell Metab. 13, 450-460 (2011).

36. Canto, C., Menzies, K. J. \& Auwerx, J. NAD metabolism and the control of energy homeostasis: a balancing act between mitochondria and the nucleus. Cell Metab. 22 31-53 (2015).

37. Bugarski, M., Martins, J. R., Haenni, D. \& Hall, A. M. Multiphoton imaging reveals axial differences in metabolic autofluorescence signals along the kidney proximal tubule. Am. J. Physiol. Renal Physiol. 315 F1613-F1625 (2018)

38. Uchida, S. \& Endou, H. Substrate specificity to maintain cellular ATP along the mouse nephron. Am. J. Physiol. 255, F977-F983 (1988)

39. Pagliarini, D. J. et al. A mitochondrial protein compendium elucidates complex I disease biology. Cell 134, 112-123 (2008).

40. Emma, F., Montini, G., Parikh, S. M. \& Salviati, L. Mitochondrial dysfunction in inherited renal disease and acute kidney injury. Nat. Rev. Nephrol. 12 267-280 (2016)

41. Shi, H. et al. NAD deficiency, congenital malformations, and niacin supplementation. N. Engl. J. Med. 377 544-552 (2017)

42. Martin, D. R., Lewington, A. J., Hammerman, M. R. \& Padanilam, B. J. Inhibition of poly(ADP-ribose) polymerase attenuates ischemic renal injury in rats. Am. J. Physiol. Regul. Integr. Comp. Physiol. 279, R1834-R1840 (2000).
43. Liu, S. B., Liu, J., Liu, D. W., Wang, X. T. \& Yang, R. L. Inhibition of poly-(ADP-ribose) polymerase protects the kidney in a canine model of endotoxic shock. Nephron 130, 281-292 (2015).

44. Zheng, J., Devalaraja-Narashimha, K., Singaravelu, K \& Padanilam, B. J. Poly(ADP-ribose) polymerase-1 gene ablation protects mice from ischemic renal injury. Am. J. Physiol. Renal Physiol. 288, F387-F398 (2005).

45. Vaschetto, R. et al. Renal hypoperfusion and impaired endothelium-dependent vasodilation in an animal model of VILI: the role of the peroxynitrite-PARP pathway. Crit. Care 14, R45 (2010)

46. Bracken, C. et al. Inhibition of PARP 1 attenuates rat renal ischemia reperfusion injury [abstract]. $J$. $A m$. Soc. Nephrol. 29, 440 (2018).

47. Tran, M. T. et al. PGC1 1 drives NAD biosynthesis linking oxidative metabolism to renal protection. Nature 531, 528-532 (2016)

48. Rasbach, K. A. \& Schnellmann, R. G. Signaling of mitochondrial biogenesis following oxidant injury. J. Biol. Chem. 282, 2355-2362 (2007).

49. Lynch, M. R. et al. TFEB-driven lysosomal biogenesis is pivotal for PGC $1 \alpha$-dependent renal stress resistance. JCl Insight 5, 126749 (2019).

50. Tran, M. et al. PGC-1 a promotes recovery after acute kidney injury during systemic inflammation in mice. J. Clin. Invest. 121, 4003-4014 (2011).

51. Dumesic, P. A. et al. An evolutionarily conserved uORF regulates $\mathrm{PGC} 1 \alpha$ and oxidative metabolism in mice, flies, bluefin tuna. Cell Metab. 30, 190-200 (2019).

52. Calvo, S. E., Pagliarini, D. J. \& Mootha, V. K. Upstream open reading frames cause widespread reduction of protein expression and are polymorphic among humans. Proc. Natl Acad. Sci. USA 106 7507-7512 (2009).

53. Rodgers, J. T. et al. Nutrient control of glucose homeostasis through a complex of PGC-1 $\alpha$ and SIRT 1 . Nature 434, 113-118 (2005)

54. Katsyuba, E. et al. De novo $\mathrm{NAD}^{+}$synthesis enhances mitochondrial function and improves health. Nature 563, 354-359 (2018).

55. Zheng, X. et al. Kynurenine 3-monooxygenase is a critical regulator of renal ischemia-reperfusion injury. Exp. Mol. Med. 51, 15 (2019)

56. Cervenka, I., Agudelo, L. Z. \& Ruas, J. L. Kynurenines: tryptophan's metabolites in exercise, inflammation, and mental health Science 357 eaaf9794 (2017)

57. Korstanje, R. et al. Loss of kynurenine 3-monooxygenase causes proteinuria. J. Am. Soc. Nephrol. 27, 3271-3277 (2016)

58. Weidemann, M. J. \& Krebs, H. A. The fuel of respiration of rat kidney cortex. Biochem. J. 112 149-166 (1969)

59. Van Slyke, D. D., Rhoads, C. P., Hiller, A. \& Alving, A. S. Relationships between urea extraction, renal blood flow, renal oxygen consumption, and diuresis. The mechanism urea excretion. Am. J. Physiol. 109 336-374 (1934).

60. Matthys, E., Patel, Y., Kreisberg, J., Stewart, J. H. \& Venkatachalam, M. Lipid alterations induced by renal ischemia: pathogenic factor in membrane damage. Kidney Int 26, 153-161 (1984).

61. Zager, R. A., Johnson, A. C. \& Hanson, S. Y. Renal tubular triglyercide accumulation following endotoxic, toxic, and ischemic injury. Kidney Int. 67, 111-121 (2005).

62. Tran, M. T. et al. PGC1 a drives NAD biosynthesis linking oxidative metabolism to renal protection. Nature 531, 528-532 (2016)

63. Kim, W. et al. Polyunsaturated fatty acid desaturation is a mechanism for glycolytic NAD+ recycling. Cell Metab. 29, 856-870.e7 (2019).

64. Guan, Y. et al. Nicotinamide mononucleotide, an NAD precursor, rescues age-associated susceptibility to AK in a sirtuin 1-dependent manner. J. Am. Soc. Nephrol. 28, 2337-2352 (2017)

65. Sardiello, M. et al. A gene network regulating lysosomal biogenesis and function. Science 325 , 473-477 (2009)

66. Settembre, C. et al. TFEB links autophagy to lysosomal biogenesis. Science 332, 1429-1433 (2011).

67. Morigi, M. et al. Sirtuin 3-dependent mitochondrial dynamic improvements protect against acute kidney injury. J. Clin. Invest. 125, 715-726 (2015).

68. Zhao, W. Y., Zhang, L., Sui, M. X., Zhu, Y. H. \& Zeng, L. Protective effects of sirtuin 3 in a murine model of sepsis-induced acute kidney injury. Sci. Rep. 6, 33201 (2016).

69. Zhou, H. L. et al. Metabolic reprogramming by the S-nitroso-CoA reductase system protects against kidney injury. Nature 565, 96-100 (2019).
70. Kirkland, J. B. Niacin requirements for genomic stability. Mutat. Res. 733, 14-20 (2012).

71. Drury, E. R. et al. Renal PGC1 a may be associated with recovery after delayed graft function. Nephron 138, 303-309 (2018)

72. Dobrovolsky, V. N. et al. Effect of arylformamidase (kynurenine formamidase) gene inactivation in mice on enzymatic activity, kynurenine pathway metabolites and phenotype. Biochim. Biophys. Acta 1724 163-172 (2005).

73. Dobrovolsky, V. N., Bucci, T., Heflich, R. H., Desjardins, J. \& Richardson, F. C. Mice deficient for cytosolic thymidine kinase gene develop fatal kidney disease. Mol. Genet. Metab. 78, 1-10 (2003).

74. Zhang, W. et al. Genome-wide association mapping of quantitative traits in outbred mice. G3 2, 167-174 (2012).

75. Zheng, M. et al. Nicotinamide reduces renal interstitial fibrosis by suppressing tubular injury and inflammation. J. Cell. Mol. Med. 23, 3995-4004 (2019)

76. Hasegawa, K. et al. Renal tubular Sirt1 attenuates diabetic albuminuria by epigenetically suppressing claudin-1 overexpression in podocytes. Nat. Med. 19 1496-1504 (2013).

77. Chen, Y. et al. Endogenous Nampt upregulation is associated with diabetic nephropathy inflammatoryfibrosis through the NF-kB p65 and Sirt1 pathway; NMN alleviates diabetic nephropathy inflammatoryfibrosis by inhibiting endogenous Nampt. Exp. Ther Med. 14, 4181-4193 (2017)

78. Dugan, L. L. et al. AMPK dysregulation promotes diabetes-related reduction of superoxide and mitochondrial function. J. Clin. Invest. 123, 4888-4899 (2013).

79. Long, J. et al. Long noncoding RNA Tug 1 regulates mitochondrial bioenergetics in diabetic nephropathy. J. Clin. Invest. 126, 4205-4218 (2016).

80. Li, S. Y. et al. Increasing the level of peroxisome proliferator-activated receptor gamma coactivator-1a in podocytes results in collapsing glomerulopathy. JCl Insight 2, 92930 (2017).

81. Kang, H. M. et al. Defective fatty acid oxidation in renal tubular epithelial cells has a key role in kidney fibrosis development. Nat. Med. 21, 37-46 (2015)

82. Han, S. H. et al. Deletion of Lkb1 in renal tubular epithelial cells leads to ckd by altering metabolism. J. Am. Soc. Nephrol. 27, 439-453 (2016).

83. Rhee, E. P. et al. A combined epidemiologic and metabolomic approach improves CKD prediction. J. Am. Soc. Nephrol. 24, 1330-1338 (2013).

84. Goek, O. N. et al. Metabolites associate with kidney function decline and incident chronic kidney disease in the general population. Nephrol. Dial. Transpl. 28 , 2131-2138 (2013).

85. Yu, B. et al. Serum metabolomic profiling and incident CKD among African Americans. Clin. J. Am. Soc. Nephrol. 9, 1410-1417 (2014).

86. Ix, J. H. et al. Effects of nicotinamide and lanthanum carbonate on serum phosphate and fibroblast growth factor-23 in CKD: the combine trial. J. Am. Soc. Nephrol. 30, 1096-1108 (2019).

87. Lin, S.-J., Defossez, P.-A. \& Guarente, L. Requirement of NAD and SIR2 for life-span extension by calorie restriction in Saccharomyces cerevisiae. Science $\mathbf{2 8 9}$ 2126-2128 (2000).

88. Gomes, A. P. et al. Declining NAD+ induces a pseudohypoxic state disrupting nuclear-mitochondria communication during aging. Cell 155, 1624-1638 (2013).

89. Braidy, $\mathrm{N}$. et al. Age related changes in NAD metabolism oxidative stress and Sirt 1 activity in wistar rats. PLOS ONE 6, e19194 (2011).

90. Zhu, X.-H., Lu, M., Lee, B.-Y., Ugurbil, K. \& Chen, W. In vivo NAD assay reveals the intracellular NAD contents and redox state in healthy human brain and their age dependences. Proc. Natl Acad. Sci. USA 112, 2876-2881 (2015)

91. Anderson, S. et al. Acute kidney injury in older adults. J. Am. Soc. Nephrol. 22, 28-38 (2011).

92. Amdur, R. L., Chawla, L. S., Amodeo, S., Kimmel, P. L. \& Palant, C. E. Outcomes following diagnosis of acute renal failure in U.S. veterans: focus on acute tubular necrosis. Kidney Int. 76, 1089-1097 (2009).

93. Cerda, J. et al. Epidemiology of acute kidney injury. Clin. J. Am. Soc. Nephrol. 3, 881-886 (2008)

94. Schmitt, R. et al. Recovery of kidney function after acute kidney injury in the elderly: a systematic review and meta-analysis. Am. J. Kidney Dis. 52, 262-271 (2008). 
95. Lindeman, R. D., Tobin, J. \& Shock, N. W. Longitudinal studies on the rate of decline in renal function with age. J. Am. Geriatr. Soc. 33, 278-285 (1985).

96. Poggio, E. D. et al. Demographic and clinical characteristics associated with glomerular filtration rates in living kidney donors. Kidney Int. 75 1079-1087 (2009)

97. Hollenberg, N. K. et al. Age, renal perfusion and function in island-dwelling indigenous Kuna Amerinds of Panama. Nephron 82, 131-138 (1999).

98. Hommos, M. S Glassock, R. J \& Rule, A. D. Structural and functional changes in human kidneys with healthy aging. J. Am. Soc. Nephrol. 28, 2838-2844 (2017)

99. Yaku, K., Okabe, K. \& Nakagawa, T. Simultaneous measurement of NAD metabolome in aged mice tissue using liquid chromatography tandem-mass spectrometry. Biomed. Chromatogr. 32, e4205 (2018).

100. Balaban, R. S. \& Mandel, L. J. Coupling of aerobic metabolism to active ion transport in the kidney. J. Physiol. 304, 331-348 (1980).

101. Harris, S. I., Balaban, R. S. \& Mandel, L. J. Oxygen consumption and cellular ion transport: evidence for adenosine triphosphate to $\mathrm{O} 2$ ratio near 6 in intact cell. Science 208, 1148-1150 (1980).

102. Gardell, S. J. et al. Boosting NAD ${ }^{+}$with a small molecule that activates NAMPT. Nat. Commun. 10, 3241 (2019).

103. Gerdts, J., Brace, E. J., Sasaki, Y., DiAntonio, A \& Milbrandt, J. SARM 1 activation triggers axon degeneration locally via $\mathrm{NAD}^{+}$destruction. Science 348, 453-457 (2015).

104. Hsu, C. P., Oka, S., Shao, D., Hariharan, N. \& Sadoshima, J. Nicotinamide phosphoribosyltransferase regulates cell survival through $\mathrm{NAD}^{+}$synthesis in cardiac myocytes. Circ. Res. 105, 481-491 (2009).

105. Yamamoto, T. et al. Nicotinamide mononucleotide, an intermediate of $\mathrm{NAD}^{+}$synthesis, protects the heart from ischemia and reperfusion. PLOS ONE 9, e98972 (2014).
106. Siegel, C. S. \& McCullough, L. D. NAD ${ }^{+}$and nicotinamide: sex differences in cerebral ischemia. Neuroscience 237, 223-231 (2013).

107. Park, J. H., Long, A., Owens, K. \& Kristian, T. Nicotinamide mononucleotide inhibits post-ischemic $\mathrm{NAD}^{+}$degradation and dramatically ameliorates brain damage following global cerebral ischemia. Neurobiol. Dis. 95, 102-110 (2016)

108. Fang, E. F. et al. NAD+ replenishment improves lifespan and healthspan in ataxia telangiectasia models via mitophagy and DNA repair. Cell. Metab. 24, 566-581 (2016).

109. Kraus, D. et al. Nicotinamide $\mathrm{N}$-methyltransferase knockdown protects against diet-induced obesity. Nature 508, 258-262 (2014).

110. Kanehisa Laboratories. Tryptophan metabolism Homo sapiens (human). KEGG https://www.genome.jp kegg-bin/show_pathway?hsa00380 (2019).

111. Pillai, V. B. et al. Exogenous NAD blocks cardiac hypertrophic response via activation of the SIRT3LKB1-AMP-activated kinase pathway. J. Biol. Chem. 285, 3133-3144 (2010)

112. Ryu, D. et al. $\mathrm{NAD}^{+}$repletion improves muscle function in muscular dystrophy and counters global PARylation. Sci. Transl Med. 8, 361 ra139 (2016).

113. Zhang, $\mathrm{H}$. et al. $\mathrm{NAD}^{+}$repletion improves mitochondrial and stem cell function and enhances life span in mice. Science 352, 1436-1443 (2016).

114. Hong, S. et al. Nicotinamide $\mathrm{N}$-methyltransferase regulates hepatic nutrient metabolism through Sirt1 protein stabilization. Nat. Med. 21, 887-894 (2015).

115. Qin, W. et al. Neuronal SIRT1 activation as a novel mechanism underlying the prevention of Alzheimer disease amyloid neuropathology by calorie restriction. J. Biol. Chem. 281, 21745-21754 (2006).

116. Lehmann, S., Loh, S. H. \& Martins, L. M. Enhancing $\mathrm{NAD}^{+}$salvage metabolism is neuroprotective in a PINK model of Parkinson's disease. Biol. Open 6, 141-147 (2017).

117. Fang, E. F. et al. NAD+ replenishment improves lifespan and healthspan in ataxia telangiectasia models via mitophagy and DNA repair. Cell Metab. 24, 566-581 (2016).

118. Wang, G. et al. P7C3 neuroprotective chemicals function by activating the rate-limiting enzyme in NAD salvage. Cell 158, 1324-1334 (2014).

119. Williams, P. A. et al. Vitamin B3 modulates mitochondrial vulnerability and prevents glaucoma in aged mice. Science 355, 756-760 (2017).

120. Chen, A. C. et al. A phase 3 randomized trial of nicotinamide for skin-cancer chemoprevention N. Engl. J. Med. 373, 1618-1626 (2015).

121. Sharma, K. et al. Metabolomics reveals signature of mitochondrial dysfunction in diabetic kidney disease. J. Am. Soc. Nephrol 24, 1901-1912 (2013).

\section{Acknowledgements}

The authors thank members of their laboratories, their scientific collaborators and colleagues in the field for fruitful discussions. They regret being unable to cite and discuss many outstanding studies owing to space constraints.

\section{Author contributions}

All authors researched the data for the article, contributed to discussions of the content, wrote the text and reviewed or edited the manuscript before submission.

\section{Competing interests}

S.M.P. is an inventor of patent filings from Beth Israel Deaconess Medical Center. He holds equity in Raksana, serves on the Scientific Advisory Board of Aerpio and has received consulting fees from Cytokinetics, Astellas, Janssen, Mission Therapeutics and Aerpio. K.M.R. and E.P.R. declare no competing interests.

\section{Peer review information}

Nature Reviews Nephrology thanks C. Brenner, R Schnellmann and the other, anonymous, reviewer(s) for their contribution to the peer review of this work.

\section{Publisher's note}

Springer Nature remains neutral with regard to jurisdictional claims in published maps and institutional affiliations. 